\title{
Search for a fermiophobic Higgs boson in the diphoton decay channel with the ATLAS detector
}

\author{
The ATLAS Collaboration ${ }^{\star}$ \\ CERN, 1211 Geneva 23, Switzerland
}

Received: 3 May 2012 / Revised: 1 August 2012

(C) CERN for the benefit of the ATLAS collaboration 2012. This article is published with open access at Springerlink.com

\begin{abstract}
A search for a fermiophobic Higgs boson using diphoton events produced in proton-proton collisions at a centre-of-mass energy of $\sqrt{s}=7 \mathrm{TeV}$ is performed using data corresponding to an integrated luminosity of $4.9 \mathrm{fb}^{-1}$ collected by the ATLAS experiment at the Large Hadron Collider. A specific benchmark model is considered where all the fermion couplings to the Higgs boson are set to zero and the bosonic couplings are kept at the Standard Model values (fermiophobic Higgs model). The largest excess with respect to the background-only hypothesis is found at $125.5 \mathrm{GeV}$, with a local significance of 2.9 standard deviations, which reduces to 1.6 standard deviations when taking into account the look-elsewhere effect. The data exclude the fermiophobic Higgs model in the ranges 110.0118.0 GeV and 119.5-121.0 GeV at $95 \%$ confidence level.
\end{abstract}

Several extensions of the Standard Model (SM) have been proposed in which the Higgs field couplings to some or all fermion generations are substantially suppressed, for example two Higgs doublet models or Higgs triplet models [1-4]. A fermiophobic benchmark model, in which the Higgs field couplings to all fermions are set to zero while the couplings to bosons are kept at their SM values, has been introduced to allow a generic investigation of these scenarios [5].

In such a model, the production of the Higgs boson in hadron colliders and its decay properties are significantly altered compared to the SM. Fermiophobic Higgs bosons can only be produced via vector boson fusion (VBF) or associated production with vector bosons $(\mathrm{VH}, \mathrm{V}=W, Z$ ). Because Higgs boson decays to fermions are absent at tree level, the branching fractions for decays to gauge bosons are enhanced. In addition, the partial width of the decay to two photons is enhanced by the suppression of the destructive interference between the $W$-boson and top-quark loops. The

^e-mail: atlas.publications@cern.ch resulting cross section times branching ratio for fermiophobic Higgs boson production with decay to two photons is larger than that of the SM for Higgs boson masses $\left(m_{H}\right)$ below $125 \mathrm{GeV}$. Table 1 lists, for several values of $m_{H}$, the fermiophobic Higgs boson cross section multiplied by the decay branching ratio into two photons. The ratio of this quantity with respect to that of the SM Higgs boson and the enhancement of the diphoton branching ratio are also shown. In addition to the enhanced diphoton decay rates, the recoiling jets or vector bosons in the VBF or VH production modes, respectively, imply a high transverse momentum for the Higgs boson that can be exploited as a discriminating variable in the analysis. However, for increasing $m_{H}$ the diphoton decay rate falls rapidly, making the search less sensitive at higher masses in this decay channel.

Searches for a fermiophobic Higgs boson have been performed at the LEP and Tevatron colliders. The combination of results from the LEP experiments [5] excludes a fermiophobic Higgs boson at $95 \%$ confidence level (CL) for masses below $109 \mathrm{GeV}$. When including both the $W W$ and $\gamma \gamma$ decay modes, the Tevatron experiments exclude a fermiophobic Higgs boson with masses up to $119 \mathrm{GeV}[6,7]$.

This letter describes a search for a fermiophobic Higgs boson using diphoton events produced in proton-proton collisions at a centre-of-mass energy of $\sqrt{s}=7 \mathrm{TeV}$ using data corresponding to an integrated luminosity of $4.9 \mathrm{fb}^{-1}$ collected by the ATLAS experiment. This analysis follows exactly that of the related search for a SM Higgs boson with the same dataset [8], but the fermiophobic Higgs hypothesis is used to construct the signal model. The sensitivity to the fermiophobic signal is larger than that for the SM Higgs due to the larger diphoton transverse momentum.

The ATLAS detector is described in detail in Ref. [9]. The most relevant subsystems for this analysis are the calorimeter, in particular the electromagnetic section, and the inner detector. The electromagnetic calorimeter is a lead-liquid-argon detector, finely segmented in the lateral 
Table 1 Higgs boson production cross section multiplied by the branching ratio into two photons for the fermiophobic benchmark model $\left(\sigma_{\mathrm{f}}\right)$, the ratio of this value to the SM value $\left(\sigma_{\mathrm{f}} / \sigma_{\mathrm{SM}}\right)$ and the two photon branching ratio enhancement compared to the $\mathrm{SM}\left(\mathcal{B}_{\mathrm{f}} / \mathcal{B}_{\mathrm{SM}}\right)$ for various fermiophobic Higgs boson masses. The expected number of signal events after candidate selection are also shown for $4.9 \mathrm{fb}^{-1}$ of data as well as the overall signal selection efficiencies

\begin{tabular}{llllllllll}
\hline$m_{H}[\mathrm{GeV}]$ & 110 & 115 & 120 & 125 & 130 & 135 & 140 & 145 & 150 \\
\hline$\sigma_{\mathrm{f}}[\mathrm{fb}]$ & 163 & 90 & 53 & 32 & 21 & 13 & 8.9 & 5.9 & 3.9 \\
$\sigma_{\mathrm{f}} / \sigma_{\mathrm{SM}}$ & 3.7 & 2.1 & 1.2 & 0.8 & 0.6 & 0.4 & 0.3 & 0.3 & 0.2 \\
$\mathcal{B}_{\mathrm{f}} / \mathcal{B}_{\mathrm{SM}}$ & 30.2 & 17.0 & 10.3 & 6.7 & 4.7 & 3.5 & 2.8 & 2.3 & 2.0 \\
Signal events & 255 & 149 & 91 & 58 & 38 & 25 & 17 & 12 & 7.9 \\
Efficiency $[\%]$ & 32 & 34 & 35 & 37 & 38 & 38 & 39 & 40 & 42 \\
\hline
\end{tabular}

and longitudinal directions. It is composed of a barrel part covering the pseudorapidity range $|\eta|<1.475$ and two endcap sections covering $1.375<|\eta|<3.2$. The barrel $(|\eta|<$ $0.8)$ and extended barrel $(0.8<|\eta|<1.7)$ hadron calorimeter sections consist of steel and scintillating tiles, while the end-cap sections $(1.5<|\eta|<3.2)$ are composed of copper and liquid argon. The inner detector includes silicon-based pixel and micro-strip detectors in the range $|\eta|<2.5$, and a transition radiation tracker with electron identification capability extending out to $|\eta|<2.0$. It is surrounded by a superconducting solenoid that provides a $2 \mathrm{~T}$ axial magnetic field.

Data used in this analysis were recorded using a diphoton trigger with a $20 \mathrm{GeV}$ transverse energy $\left(E_{\mathrm{T}}\right)$ threshold on each photon. This trigger is seeded by a first-level trigger, which requires two clusters in the electromagnetic calorimeter with $E_{\mathrm{T}}>14 \mathrm{GeV}$ or $E_{\mathrm{T}}>12 \mathrm{GeV}$, depending on the data-taking period. This trigger has a signal efficiency close to $99 \%$ following the final event selection. After application of data-quality requirements the analysed data sample corresponds to a total integrated luminosity of $4.9 \pm 0.2 \mathrm{fb}^{-1}[10,11]$.

The events are required to have at least one reconstructed vertex with a minimum of three associated tracks, where the transverse momentum of each track is required to be larger than $0.4 \mathrm{GeV}$. At least two photons within the fiducial region $|\eta|<2.37$ (excluding the transition region between the barrel and the end-cap, $1.37<|\eta|<1.52$ ) satisfying tight identification criteria based on electromagnetic shower shapes [12] are required. The transverse momenta for the leading and sub-leading photons are required to be larger than $40 \mathrm{GeV}$ and $25 \mathrm{GeV}$, respectively. The photon reconstruction and identification efficiency ranges typically from $65 \%$ to $95 \%$ for $E_{\mathrm{T}}$ in the range between $25 \mathrm{GeV}$ and $80 \mathrm{GeV}$. The transverse energy deposited around each photon within a cone of $\Delta R=\sqrt{(\Delta \eta)^{2}+(\Delta \phi)^{2}}=0.4$, excluding the deposits of the photon itself, is required to be less than $5 \mathrm{GeV}$. Corrections for the small estimated energy leakage outside the excluded region, the underlying event and effects of additional minimum bias interactions occurring in the same or neighbouring bunch crossings (in-time and out-of-time pileup) are applied to this quantity on an event-by-event basis.

The invariant mass of each diphoton candidate $\left(m_{\gamma \gamma}\right)$ is evaluated using the photon energies, the impact points measured in the calorimeter and the production vertex. The photon energy calibration is performed independently for converted and unconverted photons. Converted photons are defined to be those with a well-reconstructed conversion vertex in the inner detector. A detailed simulation of the detector geometry and response is used for the calibration. Additional corrections due to mis-modelling of the material in front of the calorimeter and of calorimeter non-uniformities are applied. These amount to about $\pm 1 \%$ depending on the pseudorapidity of the photon and are obtained from studies of $Z \rightarrow e^{+} e^{-}$decays in data [13]. The diphoton production vertex along the beam axis is determined by combining the trajectories of each photon, measured using the longitudinal segmentation of the calorimeter, with a constraint from the average beam spot position. The position of the conversion vertex is also used where the photons convert in the tracking region instrumented with silicon detectors. Conversion candidates with tracks reconstructed in inactive regions of the innermost pixel layer are rejected to reduce the contamination from misidentified electrons. The resolution of the diphoton mass reconstructed using this method is dominated by the photon energy resolution.

A total of 22,489 events were selected with a diphoton invariant mass between $100 \mathrm{GeV}$ and $160 \mathrm{GeV}$. Although not used directly in the final result, the diphoton sample composition was studied using a two-dimensional side-band technique based on photon identification quality and isolation [8]. The fraction of true diphoton events was estimated to be $(71 \pm 5) \%$. The rest of the background is due to events with one or more misidentified jets, except for a small $(\sim 0.7 \%)$ contribution from Drell-Yan events where both electrons pass the photon selection.

To enhance the sensitivity of the analysis, the data sample is split into nine categories, each with different expected signal mass resolutions, signal yields and signal-to-background ratios (S/B). This categorisation depends on the impact point of the photons on the calorimeter, the presence of photon conversions and the value of the component of the diphoton transverse momentum orthogonal to the diphoton thrust-like axis in the transverse plane ${ }^{1}\left(p_{\mathrm{Tt}}\right)[14,15]$.

Events in which both photons are unconverted are separated into the unconverted central (both photons in the cen${ }^{1} p_{\mathrm{Tt}}=\left|\mathbf{p}_{\mathrm{T}}^{\gamma \gamma} \times \widehat{t}\right|$, where $\widehat{t}=\frac{\mathbf{p}_{\mathrm{T}}^{\gamma_{1}}-\mathbf{p}_{\mathrm{T}}^{\gamma_{2}}}{\left|\mathbf{p}_{\mathrm{T}}^{\gamma_{1}}-\mathbf{p}_{\mathrm{T}}^{\gamma_{2}}\right|}$ denotes the transverse thrust,
$\mathbf{p}_{\mathrm{T}}^{\gamma_{1}}$ and $\mathbf{p}_{\mathrm{T}}^{\gamma_{2}}$ are the transverse momenta of the two photons, and
$\mathbf{p}_{\mathrm{T}}^{\gamma \gamma}=\mathbf{p}_{\mathrm{T}}^{\gamma_{1}}+\mathbf{p}_{\mathrm{T}}^{\gamma_{2}}$ is the transverse momentum of the diphoton system. 
tral region of the barrel calorimeter, $|\eta|<0.75$ ) and unconverted rest (all other events) categories. Events for which at least one photon is converted are separated into the converted central (both photons within $|\eta|<0.75$ ), converted transition (at least one photon close to the barrel/end-cap transition region, $1.3<|\eta|<1.75$ ) and converted rest (the remaining events) categories.

With the exception of the converted transition category, all the events are further subdivided into low $p_{\mathrm{Tt}}\left(p_{\mathrm{Tt}}<\right.$ $40 \mathrm{GeV}$ ) and high $p_{\mathrm{Tt}}$ (all other events) categories. Monte Carlo (MC) simulation studies show that a fermiophobic Higgs boson signal has larger $p_{\mathrm{Tt}}$ on average than background events. This quantity is strongly correlated with the diphoton transverse momentum but offers several advantages. Higher values of $p_{\mathrm{Tt}}$ do not include kinematic configurations for which the two photons are back-to-back in the azimuthal plane with substantially different transverse momenta. This reduces biases on the identification and isolation (transverse energy deposited around the photon) of the sub-leading photon in the high $p_{\mathrm{Tt}}$ categories and retains a monotonically falling diphoton invariant mass distribution for the background events at the chosen cut values. The latter quality is advantageous for the background modelling and associated uncertainties discussed below.

A full Geant 4-based [16] MC simulation [17] of Higgs boson events decaying into two photons is used to model the expected signal. The signal yields are normalised to next-to-next-to-leading-order production cross sections [1823] and the branching ratios for the fermiophobic Higgs boson are calculated using HDECAY [24]. Higgs boson VBF production is simulated using POWHEG [25] interfaced with PYTHIA [26] for showering and hadronisation, while PYTHIA is chosen for the VH processes. Pileup effects are simulated by overlaying each MC event with a variable number of simulated inelastic $p p$ collisions, taking into account the LHC bunch-train structure [27].

A set of corrections is applied to the simulated events in order to match the data-taking conditions. The simulated events are re-weighted to reproduce the distribution of the average number of interactions per bunch crossing reconstructed in the data, which has a mean value of about nine for the data sample used in this analysis. The energies of the simulated photons are smeared to account for differences observed in studies of the calorimeter resolution with $Z \rightarrow e^{+} e^{-}$decays. Calorimeter shower shapes used in the photon identification are slightly shifted to improve the agreement with the distributions observed with inclusive photons from data.

The number of fermiophobic Higgs bosons expected after candidate selection and the overall signal selection efficiency for various values of $m_{H}$ are shown in Table 1 . The signal selection efficiency increases from $32 \%$ to $42 \%$ as the Higgs boson mass increases from $110 \mathrm{GeV}$ to $150 \mathrm{GeV}$.
The signal is modelled as the sum of a core component, described by a Crystal Ball (CB) function [28], and a wider Gaussian component incorporating outlying events. The latter component typically accounts for less than $5 \%$ of the signal. Table 2 lists the expected full-width-at-half-maximum $(\mathrm{FWHM})$ and Gaussian width of the core component $\left(\sigma_{\mathrm{CB}}\right)$ for each of the nine event categories. The expected number of signal events for $m_{H}=120 \mathrm{GeV}$, the number of background events in the diphoton mass range of $100 \mathrm{GeV}$ to $160 \mathrm{GeV}$, and the signal-to-background ratio in a mass window containing $90 \%$ of the signal are also shown. The main sensitivity to the fermiophobic production modes comes from the high $p_{\mathrm{Tt}}$ categories due to their enhanced signal yields and signal-to-background ratios. Figure 1 shows the signal diphoton mass distribution summed over the high $p_{\mathrm{Tt}}$ categories for a Higgs boson mass of $120 \mathrm{GeV}$.

The observed diphoton invariant mass distribution in each category is modelled by an exponential function. A fit to the data is performed for which the slope and normalisation are unconstrained. Studies with large samples of simulated diphoton events show that this simple function gives a good description of the expected shape. The small systematic uncertainties associated with this assumption are discussed below. Figures 2(a) and 2(b) show the diphoton mass distributions of the selected data events summed over the low and high $p_{\mathrm{Tt}}$ categories, respectively. The converted transition category is included in the low $p_{\mathrm{Tt}}$ categories.

Systematic uncertainties affecting the signal significance arise from uncertainties on the predicted signal yields, the expected partition of the signal among the categories and the modelling of the signal and background shapes. The dominant experimental uncertainty on the signal yield is due to the imperfect knowledge of the photon reconstruction and

Table 2 Expected signal mass resolution $\left(\sigma_{\mathrm{CB}}\right.$ and FWHM in $\mathrm{GeV}$, see text) and total number of signal events $\left(N_{\mathrm{S}}\right)$ for $m_{H}=120 \mathrm{GeV}$ for each of the nine analysis categories and for the inclusive case. Also shown for each category are the number of observed events $\left(N_{\mathrm{D}}\right)$ in the diphoton mass range from $100 \mathrm{GeV}$ to $160 \mathrm{GeV}$, and the expected signal-to-background ratio (S/B) in a mass window containing $90 \%$ of the signal

\begin{tabular}{lllrrr}
\hline Category & $\sigma_{\mathrm{CB}}$ & FWHM & \multicolumn{1}{l}{$N_{\mathrm{S}}$} & \multicolumn{1}{l}{$N_{\mathrm{D}}$} & $\mathrm{S} / \mathrm{B}$ \\
\hline Unconverted central, low $p_{\mathrm{Tt}}$ & 1.4 & 3.3 & 6.2 & 1763 & 0.03 \\
Unconverted central, high $p_{\mathrm{Tt}}$ & 1.3 & 3.2 & 8.6 & 235 & 0.37 \\
Unconverted rest, low $p_{\mathrm{Tt}}$ & 1.7 & 3.9 & 12.1 & 6234 & 0.02 \\
Unconverted rest, high $p_{\mathrm{Tt}}$ & 1.6 & 3.8 & 16.0 & 1006 & 0.13 \\
Converted central, low $p_{\mathrm{Tt}}$ & 1.6 & 3.8 & 4.0 & 1318 & 0.02 \\
Converted central, high $p_{\mathrm{Tt}}$ & 1.5 & 3.5 & 5.8 & 184 & 0.26 \\
Converted rest, low $p_{\mathrm{Tt}}$ & 2.0 & 4.6 & 11.8 & 7311 & 0.01 \\
Converted rest, high $p_{\mathrm{Tt}}$ & 1.9 & 4.4 & 16.1 & 1072 & 0.09 \\
Converted transition & 2.3 & 5.8 & 10.8 & 3366 & 0.01 \\
\hline All categories & 1.7 & 3.9 & 91.2 & 22489 & 0.03 \\
\hline
\end{tabular}




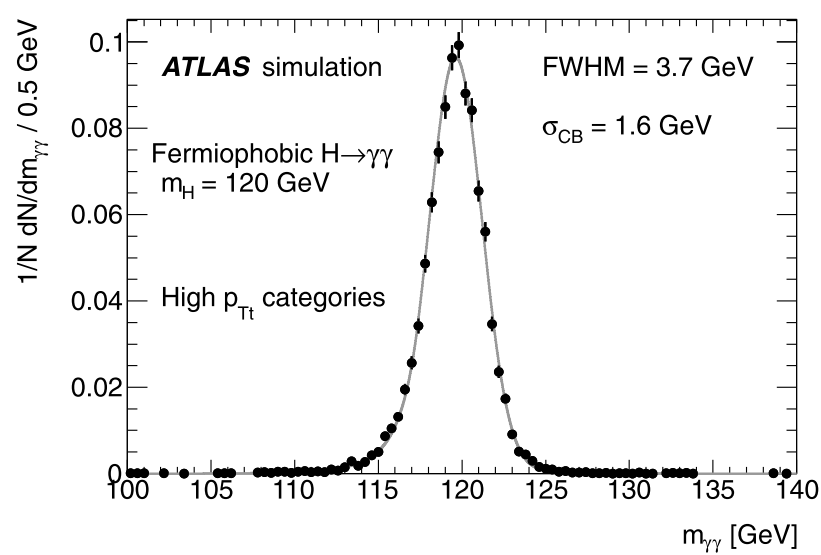

Fig. 1 Diphoton invariant mass spectrum from simulated signal samples (dots) with $m_{H}=120 \mathrm{GeV}$ summed over the high $p_{\mathrm{Tt}}$ categories, superimposed with the signal model (line)

identification efficiencies, which is estimated to be $\pm 11 \%$. This uncertainty is studied with electrons from $W$ and $Z$ boson decays in data, and photons from radiative decays of $Z$ bosons to electron and muon pairs. In addition, the effect of pileup on photon identification gives a further contribution to the signal yield uncertainty of $\pm 4 \%$. Uncertainties related to the trigger efficiency $( \pm 1 \%)$, isolation cut efficiency $( \pm 5 \%)$ and luminosity $( \pm 3.9 \%)$ are also included here.

Uncertainties on the signal cross section include a combination of the uncertainties on the parton distribution functions $[29,30]$ and $\alpha_{s}$, and uncertainties on the QCD scale. Combining the VBF and VH production modes this uncertainty is within $\pm 4 \%$ over the considered mass range. To this uncertainty, that due to the $H \rightarrow \gamma \gamma$ branching ratio $( \pm 5 \%)$ is added linearly, based on the SM calculation [23]. This yields uncertainties of $\pm 9 \%$ on the theoretical signal yield, leading to an overall uncertainty of $\pm 16 \%$ on the total signal expectation. In addition, the uncertainty on the Higgs boson $p_{\mathrm{T}}$ modelling is estimated by comparing signal samples from alternative MC generators-HERWIG [31] for VBF and ResBos [32] for VH. The result is a $\pm 1 \%$ signal migration between the low and high $p_{\mathrm{Tt}}$ categories with a negligible effect on the signal selection efficiency.

The dominant uncertainties on the signal mass resolution are due to the uncertainty on the calorimeter energy resolution $( \pm 12 \%)$ and photon calibration $( \pm 6 \%)$, which are both extrapolated from the uncertainty on the electron calibration determined using $Z$ and $J / \psi$ data [13]. The latter comes from the imperfect knowledge of the material in front of the active part of the calorimeter and is estimated using simulations with different amounts of material. This quantity also affects the fraction of expected events in the categories with converted photons; the maximal migration between converted and unconverted categories is estimated to be $\pm 4.5 \%$. Other effects on the signal mass resolution are

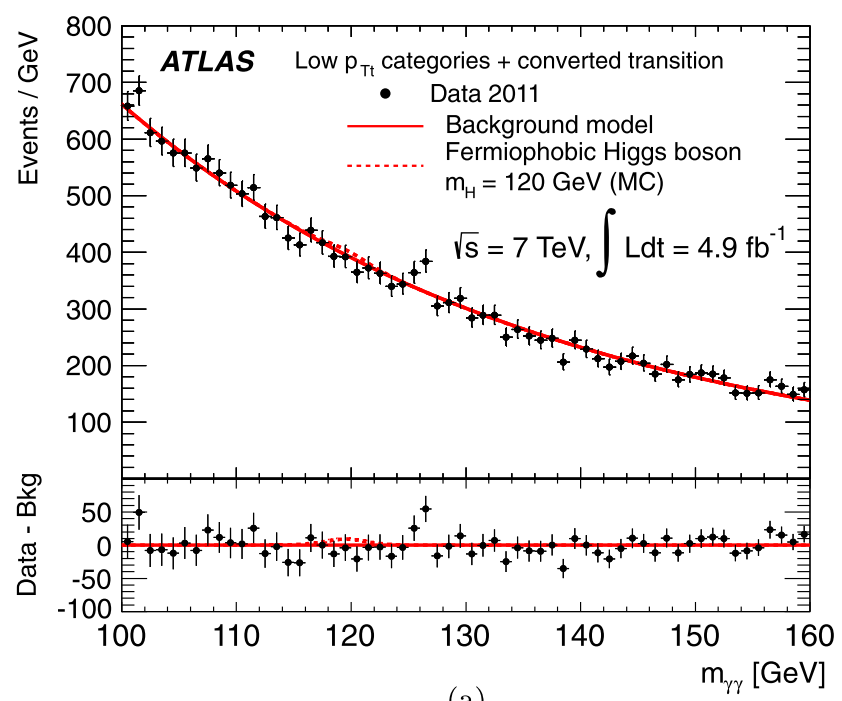

(a)

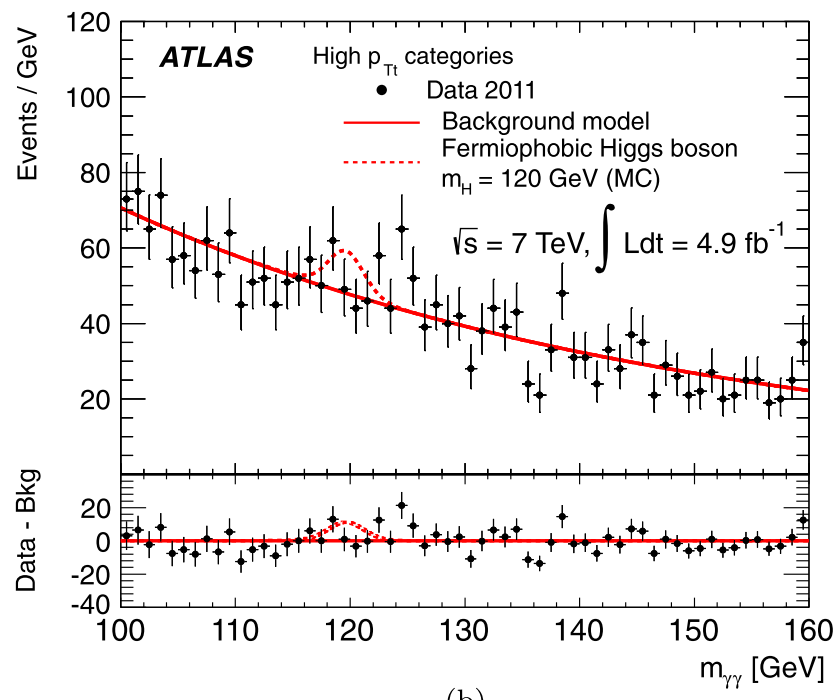

(b)

Fig. 2 Diphoton invariant mass spectra for the (a) low and (b) high $p_{\mathrm{Tt}}$ categories, overlaid with the sum of the background-only fits from the individual categories. The bottom plots show the residual of the data with respect to the fitted background. The signal expectation for a fermiophobic Higgs boson with a mass of $120 \mathrm{GeV}$ superimposed on the background fit is also shown

due to pileup fluctuations contributing to the cluster energy measurement $( \pm 3 \%)$ and to the uncertainty on the photon angular resolution $( \pm 1 \%)$ which is studied in $Z \rightarrow e^{+} e^{-}$ decays using the track-based direction measurement. The total relative uncertainty on the diphoton invariant mass resolution is thus $\pm 14 \%$.

Systematic uncertainties on the background modelling arise from a possible deviation of the background mass distribution from the assumed exponential shape. This uncertainty is evaluated as the number of events that could be mistakenly attributed to the signal. It is estimated from the adequacy of the chosen background model's description of the mass distribution predicted by ResBos [33]. The residuals 
of the fit of the background model to the ResBos diphoton mass distribution are integrated over a sliding mass window of $4 \mathrm{GeV}$, the approximate FWHM of the expected signal. The largest deviations were found at small invariant masses and these uncertainties are then applied over the whole mass range. The resulting uncertainties range from \pm 0.1 to \pm 7.9 events in the individual analysis categories, where the magnitude of these uncertainties is roughly proportional to the number of background events in each category. These absolute uncertainties do not scale with the signal strength in the final likelihood fit. For a fermiophobic Higgs boson with $m_{H}=120 \mathrm{GeV}$ the background modelling uncertainty in the high $p_{\mathrm{Tt}}$ categories is equivalent to up to $5 \%$ of the signal yield with nominal signal strength. The estimation of the uncertainties is cross-checked by fitting the data with different functional forms and comparing the result to the exponential fit.

The possible presence of a signal is investigated using a combined likelihood function constructed from the signal and background models for the diphoton invariant mass distribution in each of the nine categories. Unbinned maximum likelihood fits of the signal strength are performed, treating the systematic uncertainties as nuisance parametersfourteen in total. These nuisance parameters are added to the signal likelihood function using a Gaussian term for the background modelling uncertainty, and log-normal terms for all other uncertainties.

The compatibility of the data with the background-only hypothesis, relative to the hypothesis of background plus the fermiophobic model signal, is quantified by the local significance $p_{0}$. Figure 3 shows the result for $m_{H}$ ranging from $110 \mathrm{GeV}$ to $150 \mathrm{GeV}$, where $p_{0}$ is computed in $0.5 \mathrm{GeV}$

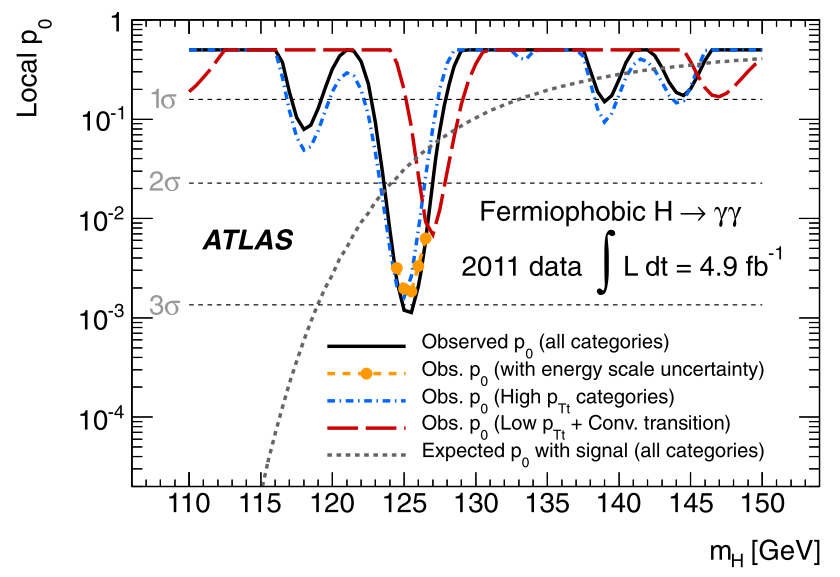

Fig. 3 Local observed $p_{0}$ as a function of the Higgs boson mass $m_{H}$ (solid line) and the median expectation for a fermiophobic signal with the given $m_{H}$ (dotted line). The five points near $125 \mathrm{GeV}$ show the observed $p_{0}$ when the uncertainty on the photon energy scale is considered. The individual contributions of the low $p_{\mathrm{Tt}}$ and high $p_{\mathrm{Tt}}$ categories to the observed $p_{0}$ are also shown steps using asymptotic formulae [34]. The contributions to $p_{0}$ values from the high $p_{\mathrm{Tt}}$ and low $p_{\mathrm{Tt}}$ categories are shown separately. The high $p_{\mathrm{Tt}}$ contribution has a minimum $p_{0}$ at $125 \mathrm{GeV}$, while the low $p_{\mathrm{Tt}}$ contribution has a minimum at $127 \mathrm{GeV}$. The larger signal-to-background ratio as well as the larger expected signal yield in the high $p_{\mathrm{Tt}}$ category compared to the low $p_{\mathrm{Tt}}$ category results in the high $p_{\mathrm{Tt}}$ contribution dominating in the final result. The combined $p_{0}$ has a minimum at $125.5 \mathrm{GeV}$ corresponding to 3.0 standard deviations. The figure also shows the $p_{0}$ value expected for a fermiophobic Higgs boson signal, as a function of Higgs boson mass.

To obtain the final result, the impact of the uncertainties on the photon energy scale is considered for Higgs boson masses in the region of the minimum $p_{0}$, as shown in Fig. 3. The corresponding effect on the measured $p_{0}$ value is estimated using pseudo-experiments, since asymptotic formulae were found not to yield accurate estimates of the probability in this case. The position of the minimum $p_{0}$ is almost unchanged and the significance is lowered to 2.9 standard deviations. Taking the look-elsewhere effect [35] into account in the range $110-150 \mathrm{GeV}$, the significance reduces to about 1.6 standard deviations, with $p_{0} \approx 0.051$. This may be compared to the result of a search for the SM Higgs boson performed with the same dataset and candidate selection [8], yielding a minimum $p_{0}$ at a mass of $126.5 \mathrm{GeV}$ with a global significance of 1.5 standard deviations. No statistically significant preference for either the SM or fermiophobic Higgs boson is observed.

Given the lack of evidence for a signal, mass-dependent exclusion limits on the fermiophobic benchmark model are calculated at the $95 \%$ confidence level (CL) with a profile likelihood ratio test statistic in the $C L_{s}$ modified frequentist approach [34, 36, 37] and are shown in Fig. 4. Fermiophobic Higgs boson masses from $110.0 \mathrm{GeV}$ to $118.0 \mathrm{GeV}$ and from $119.5 \mathrm{GeV}$ to $121.0 \mathrm{GeV}$ are excluded, while the expected exclusion mass range is $110.0-123.5 \mathrm{GeV}$. These results give more stringent lower mass limits than the previous results from LEP (108.2 GeV) [5] and the Tevatron (112.9 GeV from D0, $114 \mathrm{GeV}$ from CDF) $[6,38]$ in the diphoton decay channel.

Acknowledgements We thank CERN for the very successful operation of the LHC, as well as the support staff from our institutions without whom ATLAS could not be operated efficiently.

We acknowledge the support of ANPCyT, Argentina; YerPhI, Armenia; ARC, Australia; BMWF, Austria; ANAS, Azerbaijan; SSTC, Belarus; CNPq and FAPESP, Brazil; NSERC, NRC and CFI, Canada; CERN; CONICYT, Chile; CAS, MOST and NSFC, China; COLCIENCIAS, Colombia; MSMT CR, MPO CR and VSC CR, Czech Republic; DNRF, DNSRC and Lundbeck Foundation, Denmark; EPLANET and ERC, European Union; IN2P3-CNRS, CEA-DSM/IRFU, France; GNAS, Georgia; BMBF, DFG, HGF, MPG and AvH Foundation, Germany; GSRT, Greece; ISF, MINERVA, GIF, DIP and Benoziyo Center, Israel; INFN, Italy; MEXT and JSPS, Japan; CNRST, Morocco; FOM and NWO, Netherlands; RCN, Norway; MNiSW, Poland; GRICES 


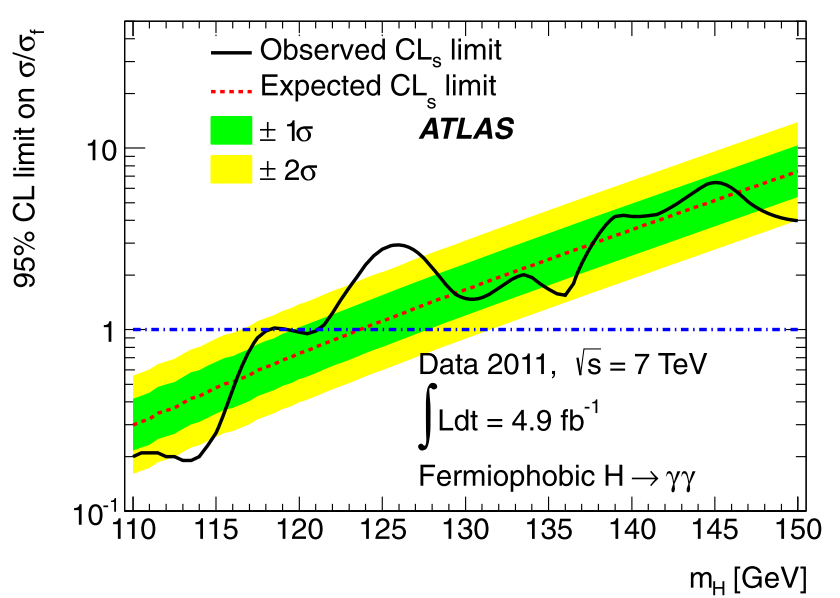

Fig. 4 Observed (solid line) and expected (dotted line) $95 \%$ CL exclusion limits for a fermiophobic Higgs boson normalised to the fermiophobic cross section times branching ratio expectation $\left(\sigma_{\mathrm{f}}\right)$ as a function of the Higgs boson mass hypothesis $\left(m_{H}\right)$

and FCT, Portugal; MERYS (MECTS), Romania; MES of Russia and ROSATOM, Russian Federation; JINR; MSTD, Serbia; MSSR, Slovakia; ARRS and MVZT, Slovenia; DST/NRF, South Africa; MICINN, Spain; SRC and Wallenberg Foundation, Sweden; SER, SNSF and Cantons of Bern and Geneva, Switzerland; NSC, Taiwan; TAEK, Turkey; STFC, the Royal Society and Leverhulme Trust, United Kingdom; DOE and NSF, United States of America.

The crucial computing support from all WLCG partners is acknowledged gratefully, in particular from CERN and the ATLAS Tier-1 facilities at TRIUMF (Canada), NDGF (Denmark, Norway, Sweden), CC-IN2P3 (France), KIT/GridKA (Germany), INFN-CNAF (Italy), NL-T1 (Netherlands), PIC (Spain), ASGC (Taiwan), RAL (UK) and BNL (USA) and in the Tier-2 facilities worldwide.

Open Access This article is distributed under the terms of the Creative Commons Attribution License which permits any use, distribution, and reproduction in any medium, provided the original author(s) and the source are credited.

\section{References}

1. P. Bamert, Z. Kunszt, Phys. Lett. B 306, 335 (1993)

2. A. Barroso, L. Brucher, R. Santos, Phys. Rev. D 60, 035005 (1999)

3. J. Gunion, R. Vega, J. Wudka, Phys. Rev. D 42, 1673 (1990)

4. A. Akeroyd, M.A. Diaz, M.A. Rivera, D. Romero, Phys. Rev. D 83, 095003 (2011)
5. LEP Higgs Working Group, ALEPH Collaboration, DELPHI Collaboration, L3 Collaboration, OPAL Collaboration, hep-ex/0107035 (2001)

6. V. Abazov et al., Phys. Rev. Lett. 107, 151801 (2011)

7. Tevatron New Higgs Working Group, CDF Collaboration, D0 Collaboration, arXiv:1109.0576 [hep-ex] (2011)

8. ATLAS Collaboration, Phys. Rev. Lett. 108, 111803 (2012)

9. ATLAS Collaboration, J. Instrum. 3, S08003 (2008)

10. ATLAS Collaboration, Eur. Phys. J. C 71, 1630 (2011)

11. ATLAS-CONF-2011-116 (2011). http://cdsweb.cern.ch/record/ 1376384

12. ATLAS Collaboration, Phys. Rev. D 83, 052005 (2011)

13. ATLAS Collaboration, Eur. Phys. J. C 72, 1909 (2012)

14. K. Ackerstaff et al., Eur. Phys. J. C 4, 47 (1998)

15. M. Vesterinen, T. Wyatt, Nucl. Instrum. Methods A 602, 432 (2009)

16. S. Agostinelli et al. (GEANT4 Collaboration), Nucl. Instrum. Methods A 506, 250 (2003)

17. ATLAS Collaboration, Eur. Phys. J. C 70, 823 (2010)

18. M. Ciccolini, A. Denner, S. Dittmaier, Phys. Rev. Lett. 99, 161803 (2007)

19. M. Ciccolini, A. Denner, S. Dittmaier, Phys. Rev. D 77, 013002 (2008)

20. P. Bolzoni, F. Maltoni, S.O. Moch, M. Zaro, Phys. Rev. Lett. 105, 011801 (2010)

21. M. Ciccolini, S. Dittmaier, M. Kramer, Phys. Rev. D 68, 073003 (2003)

22. O. Brein, A. Djouadi, R. Harlander, Phys. Lett. B 579, 149 (2004)

23. S. Dittmaier, C. Mariotti, G. Passarino, R. Tanaka (LHC Higgs Cross Section Working Group) (eds.), CERN-2011-002 (CERN, Geneva, 2011)

24. A. Djouadi, J. Kalinowski, M. Spira, Comput. Phys. Commun. 108, 56 (1998)

25. P. Nason, C. Oleari, J. High Energy Phys. 1002, 037 (2010)

26. T. Sjostrand, S. Mrenna, P.Z. Skands, J. High Energy Phys. 0605, $026(2006)$

27. ATLAS Collaboration, ATL-PHYS-PUB-2011-009 (2011). http:// cdsweb.cern.ch/record/1363300

28. J.E. Gaiser PhD. Thesis, SLAC-R-255, 1982

29. M. Botje, J. Butterworth, A. Cooper-Sarkar, A. de Roeck, J. Feltesse et al., arXiv:1101.0538 [hep-ph] (2011)

30. S. Alekhin, S. Alioli, R.D. Ball, V. Bertone, J. Blumlein et al., arXiv:1101.0536 [hep-ph] (2011)

31. G. Corcella et al., hep-ph/0210213 (2002)

32. C. Balazs, C. Yuan, Phys. Rev. D 56, 5558 (1997)

33. C. Balazs, E.L. Berger, P.M. Nadolsky, C.P. Yuan, Phys. Rev. D 76, 013009 (2007)

34. G. Cowan, K. Cranmer, E. Gross, O. Vitells, Eur. Phys. J. C 71, $1554(2011)$

35. E. Gross, O. Vitells, Eur. Phys. J. C 70, 525 (2010)

36. A.L. Read, J. Phys. G 28, 2693 (2002)

37. ATLAS and CMS Collaborations, LHC Higgs Combination Group, ATL-PHYS-PUB-2011-011 (2011). http://cdsweb.cern. $\mathrm{ch} /$ record/1375842

38. T. Aaltonen et al., Phys. Rev. Lett. 103, 061803 (2009)

\section{The ATLAS Collaboration}

G. Aad $^{48}$, B. Abbott ${ }^{111}$, J. Abdallah ${ }^{11}$, S. Abdel Khalek ${ }^{115}$, A.A. Abdelalim ${ }^{49}$, O. Abdinov ${ }^{10}$, B. Abi ${ }^{112}$, M. Abolins ${ }^{88}$, O.S. AbouZeid ${ }^{158}$, H. Abramowicz ${ }^{153}$, H. Abreu ${ }^{136}$, E. Acerbi ${ }^{89 a, 89 b}$, B.S. Acharya ${ }^{164 a, 164 b}$, L. Adamczyk ${ }^{37}$, D.L. Adams ${ }^{24}$, 
T.N. Addy ${ }^{56}$, J. Adelman ${ }^{176}$, S. Adomeit ${ }^{98}$, P. Adragna ${ }^{75}$, T. Adye ${ }^{129}$, S. Aefsky ${ }^{22}$, J.A. Aguilar-Saavedra ${ }^{124 b, a}$, M. Aharrouche $^{81}$, S.P. Ahlen ${ }^{21}$, F. Ahles ${ }^{48}$, A. Ahmad ${ }^{148}$, M. Ahsan ${ }^{40}$, G. Aielli1 ${ }^{133 a, 133 b}$, T. Akdogan ${ }^{18 a}$, T.P.A. Åkesson ${ }^{79}$, G. Akimoto $^{155}$, A.V. Akimov ${ }^{94}$, A. Akiyama ${ }^{66}$, M.S. Alam ${ }^{1}$, M.A. Alam $^{76}$, J. Albert ${ }^{169}$, S. Albrand ${ }^{55}$, M. Aleksa ${ }^{29}$, I.N. Aleksandrov $^{64}$, F. Alessandria ${ }^{89 a}$, C. Alexa ${ }^{25 a}$, G. Alexander ${ }^{153}$, G. Alexandre ${ }^{49}$, T. Alexopoulos ${ }^{9}$, M. Alhroob ${ }^{164 a, 164 c}$, M. Aliev $^{15}$, G. Alimonti ${ }^{89 a}$, J. Alison ${ }^{120}$, B.M.M. Allbrooke ${ }^{17}$, P.P. Allport ${ }^{73}$, S.E. Allwood-Spiers ${ }^{53}$, J. Almond ${ }^{82}$, A. Aloisio ${ }^{102 a, 102 b}$, R. Alon ${ }^{172}$, A. Alonso ${ }^{79}$, B. Alvarez Gonzalez ${ }^{88}$, M.G. Alviggi 102a,102b , K. Amako ${ }^{65}$, C. Amelung ${ }^{22}$, V.V. Ammosov ${ }^{128}$, A. Amorim $^{124 a, b}$, N. Amram $^{153}$, C. Anastopoulos ${ }^{29}$, L.S. Ancu $^{16}$, N. Andari ${ }^{115}$, T. Andeen $^{34}$, C.F. Anders ${ }^{58 b}$, G. Anders ${ }^{58 a}$, K.J. Anderson ${ }^{30}$, A. Andreazza ${ }^{89 a, 89 b}$, V. Andrei ${ }^{58 a}$, X.S. Anduaga ${ }^{70}$, P. Anger ${ }^{43}$, A. Angerami ${ }^{34}$, F. Anghinolfi $^{29}$, A. Anisenkov ${ }^{107}$, N. Anjos ${ }^{124 a}$, A. Annovi ${ }^{47}$, A. Antonaki ${ }^{8}$, M. Antonelli ${ }^{47}$, A. Antonov ${ }^{96}$, J. Antos ${ }^{144 b}$, F. Anulli $^{132 a}$, S. Aoun ${ }^{83}$, L. Aperio Bella ${ }^{4}$, R. Apolle ${ }^{118, c}$, G. Arabidze ${ }^{88}$, I. Aracena ${ }^{143}$, Y. Arai ${ }^{65}$, A.T.H. Arce ${ }^{44}$, S. Arfaoui $^{148}$, J-F. Arguin ${ }^{14}$, E. Arik ${ }^{18 a, *}$, M. Arik ${ }^{18 a}$, A.J. Armbruster ${ }^{87}$, O. Arnaez ${ }^{81}$, V. Arnal ${ }^{80}$, C. Arnault ${ }^{115}$, A. Artamonov $^{95}$, G. Artoni ${ }^{132 a, 132 b}$, D. Arutinov ${ }^{20}$, S. Asai ${ }^{155}$, R. Asfandiyarov ${ }^{173}$, S. Ask ${ }^{27}$, B. Åsman ${ }^{146 a, 146 b}$, L. Asquith ${ }^{5}$, K. Assamagan ${ }^{24}$, A. Astbury ${ }^{169}$, B. Aubert ${ }^{4}$, E. Auge ${ }^{115}$, K. Augsten ${ }^{127}$, M. Aurousseau ${ }^{145 a}$, G. Avolio ${ }^{163}$, R. Avramidou ${ }^{9}$, D. Axen ${ }^{168}$, G. Azuelos ${ }^{93, d}$, Y. Azuma ${ }^{155}$, M.A. Baak ${ }^{29}$, G. Baccaglionis ${ }^{89 a}$, C. Bacci ${ }^{134 a, 134 b}$, A.M. Bach ${ }^{14}$, H. Bachacou $^{136}$, K. Bachas $^{29}$, M. Backes ${ }^{49}$, M. Backhaus ${ }^{20}$, E. Badescu25a, P. Bagnaia ${ }^{132 a, 132 b}$, S. Bahinipati ${ }^{2}$, Y. Bai ${ }^{32 a}$, D.C. Bailey $^{158}$, T. Bain ${ }^{158}$, J.T. Baines ${ }^{129}$, O.K. Baker ${ }^{176}$, M.D. Baker ${ }^{24}$, S. Baker ${ }^{77}$, E. Banas ${ }^{38}$, P. Banerjee ${ }^{93}$, Sw. Banerjee ${ }^{173}$, D. Banfi ${ }^{29}$, A. Bangert ${ }^{150}$, V. Bansal ${ }^{169}$, H.S. Bansi1 ${ }^{17}$, L. Barak ${ }^{172}$, S.P. Baranov ${ }^{94}$, A. Barbaro Galtieri ${ }^{14}$, T. Barber $^{48}$, E.L. Barberio ${ }^{86}$, D. Barberis ${ }^{50,50 b}$, M. Barbero ${ }^{20}$, D.Y. Bardin ${ }^{64}$, T. Barillari ${ }^{99}$, M. Barisonzi ${ }^{175}$, T. Barklow ${ }^{143}$, N. Barlow ${ }^{27}$, B.M. Barnett ${ }^{129}$, R.M. Barnett ${ }^{14}$, A. Baroncelli ${ }^{134 a}$, G. Barone ${ }^{49}$, A.J. Barr ${ }^{118}$, F. Barreiro ${ }^{80}$, J. Barreiro ${\text { Guimarães da } \operatorname{Costa}^{57} \text {, P. Barrillon }}^{115}$, R. Bartoldus ${ }^{143}$, A.E. Barton ${ }^{71}$, V. Bartsch ${ }^{149}$, R.L. Bates ${ }^{53}$, L. Batkova ${ }^{144 a}$, J.R. Batley $^{27}$, A. Battaglia ${ }^{16}$, M. Battistin ${ }^{29}$, F. Bauer ${ }^{136}$, H.S. Bawa ${ }^{143, \text { e }, ~ S . ~ B e a l e ~}{ }^{98}$, T. Beau ${ }^{78}$, P.H. Beauchemin ${ }^{161}$, R. Beccherle $^{50 a}$, P. Bechtle ${ }^{20}$, H.P. Beck ${ }^{16}$, A.K. Becker ${ }^{175}$, S. Becker ${ }^{98}$, M. Beckingham ${ }^{138}$, K.H. Becks ${ }^{175}$, A.J. Beddall ${ }^{18 c}$, A. Beddall ${ }^{18 c}$, S. Bedikian ${ }^{176}$, V.A. Bednyakov ${ }^{64}$, C.P. Bee ${ }^{83}$, M. Begel ${ }^{24}$, S. Behar Harpaz ${ }^{152}$, M. Beimforde ${ }^{99}$, C. BelangerChampagne $^{85}$, P.J. Bell ${ }^{49}$, W.H. Bell ${ }^{49}$, G. Bella ${ }^{153}$, L. Bellagamba ${ }^{19 a}$, F. Bellina ${ }^{29}$, M. Bellomo ${ }^{29}$, A. Belloni ${ }^{57}$, O. Beloborodova $^{107, f}$, K. Belotskiy ${ }^{96}$, O. Beltramello ${ }^{29}$, O. Benary ${ }^{153}$, D. Benchekroun ${ }^{135 a}$, K. Bendtz ${ }^{146 a, 146 b}$, N. Benekos ${ }^{165}$, Y. Benhammou ${ }^{153}$, E. Benhar Noccioli ${ }^{49}$, J.A. Benitez Garcia ${ }^{159 b}$, D.P. Benjamin ${ }^{44}$, M. Benoit ${ }^{115}$, J.R. Bensinger ${ }^{22}$, K. Benslama ${ }^{130}$, S. Bentvelsen ${ }^{105}$, D. Berge ${ }^{29}$, E. Bergeaas Kuutmann ${ }^{41}$, N. Berger ${ }^{4}$, F. Berghaus ${ }^{169}$, E. Berglund ${ }^{105}$, J. Beringer ${ }^{14}$, P. Bernat ${ }^{77}$, R. Bernhard ${ }^{48}$, C. Bernius ${ }^{24}$, T. Berry ${ }^{76}$, C. Bertella ${ }^{83}$, A. Bertin ${ }^{19 a, 19 b}$, F. Bertolucci ${ }^{122 a, 122 b}$, M.I. Besana ${ }^{89 a, 89 b}$, G.J. Besjes ${ }^{104}$, N. Besson ${ }^{136}$, S. Bethke ${ }^{99}$, W. Bhimji ${ }^{45}$, R.M. Bianchi ${ }^{29}$, M. Bianco ${ }^{72 a, 72 b}$, O. Biebel ${ }^{98}$, S.P. Bieniek ${ }^{77}$, K. Bierwagen ${ }^{54}$, J. Biesiada ${ }^{14}$, M. Biglietti ${ }^{134 a}$, H. Bilokon ${ }^{47}$, M. Bindi ${ }^{19 a, 19 b}$, S. Binet ${ }^{115}$, A. Bingul ${ }^{18 c}$, C. Bini ${ }^{132 a, 132 b}$, C. Biscarat ${ }^{178}$, U. Bitenc ${ }^{48}$, K.M. Black ${ }^{21}$, R.E. Blair ${ }^{5}$, J.-B. Blanchard ${ }^{136}$, G. Blanchot ${ }^{29}$, T. Blazek ${ }^{144 a}$, C. Blocker ${ }^{22}$, J. Blocki ${ }^{38}$, A. Blondel ${ }^{49}$, W. Blum ${ }^{81}$, U. Blumenschein ${ }^{54}$, G.J. Bobbink ${ }^{105}$, V.B. Bobrovnikov ${ }^{107}$, S.S. Bocchetta $^{79}$, A. Bocci ${ }^{44}$, C.R. Boddy ${ }^{118}$, M. Boehler ${ }^{41}$, J. Boek ${ }^{175}$, N. Boelaert ${ }^{35}$, J.A. Bogaerts ${ }^{29}$, A. Bogdanchikov ${ }^{107}$, A. Bo-

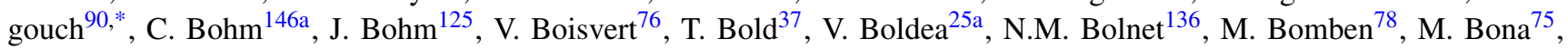
M. Bondioli ${ }^{163}$, M. Boonekamp ${ }^{136}$, C.N. Booth ${ }^{139}$, S. Bordoni ${ }^{78}$, C. Borer ${ }^{16}$, A. Borisov ${ }^{128}$, G. Borissov ${ }^{71}$, I. Borjanovic ${ }^{12 a}$, M. Borri ${ }^{82}$, S. Borroni ${ }^{87}$, V. Bortolotto ${ }^{134 a, 134 b}$, K. Bos ${ }^{105}$, D. Boscherini ${ }^{19 a}$, M. Bosman ${ }^{11}$, H. Boterenbrood ${ }^{105}$, D. Botterill $^{129}$, J. Bouchami ${ }^{93}$, J. Boudreau ${ }^{123}$, E.V. Bouhova-Thacker ${ }^{71}$, D. Boumediene ${ }^{33}$, C. Bourdarios ${ }^{115}$, N. Bousson ${ }^{83}$, A. Boveia ${ }^{30}$, J. Boyd ${ }^{29}$, I.R. Boyko ${ }^{64}$, I. Bozovic-Jelisavcic ${ }^{12 b}$, J. Bracinik ${ }^{17}$, P. Branchini ${ }^{134 a}$, A. Brandt ${ }^{7}$, G. Brandt ${ }^{118}$, O. Brandt ${ }^{54}$, U. Bratzler ${ }^{156}$, B. Brau ${ }^{84}$, J.E. Brau ${ }^{114}$, H.M. Braun ${ }^{175}$, B. Brelier ${ }^{158}$, J. Bremer ${ }^{29}$, K. Brendlinger ${ }^{120}$, R. Brenner $^{166}$, S. Bressler ${ }^{172}$, D. Britton ${ }^{53}$, F.M. Brochu ${ }^{27}$, I. Brock ${ }^{20}$, R. Brock ${ }^{88}$, E. Brodet ${ }^{153}$, F. Broggi ${ }^{89 a}$, C. Bromberg ${ }^{88}$, J. Bronner ${ }^{99}$, G. Brooijmans ${ }^{34}$, T. Brooks ${ }^{76}$, W.K. Brooks ${ }^{31 b}$, G. Brown ${ }^{82}$, H. Brown ${ }^{7}$, P.A. Bruckman de Renstrom ${ }^{38}$, D. Bruncko ${ }^{144 b}$, R. Bruneliere ${ }^{48}$, S. Brunet ${ }^{60}$, A. Bruni ${ }^{19 a}$, G. Bruni ${ }^{19 a}$, M. Bruschi ${ }^{19 a}$, T. Buanes ${ }^{13}$, Q. Buat ${ }^{55}$, F. Bucci ${ }^{49}$, J. Buchanan ${ }^{118}$, P. Buchholz ${ }^{141}$, R.M. Buckingham ${ }^{118}$, A.G. Buckley ${ }^{45}$, S.I. Buda ${ }^{25 a}$, I.A. Budagov ${ }^{64}$, B. Budick ${ }^{108}$, V. Büscher ${ }^{81}$, L. Bugge ${ }^{117}$, O. Bulekov ${ }^{96}$, A.C. Bundock ${ }^{73}$, M. Bunse ${ }^{42}$, T. Buran ${ }^{117}$, H. Burckhart ${ }^{29}$, S. Burdinn ${ }^{73}$, T. Burgess ${ }^{13}$, S. Burke ${ }^{129}$, E. Busato ${ }^{33}$, P. Bussey ${ }^{53}$, C.P. Buszello ${ }^{166}$, B. Butler ${ }^{143}$, J.M. Butler ${ }^{21}$, C.M. Buttar ${ }^{53}$, J.M. Butterworth $^{77}$, W. Buttinger ${ }^{27}$, S. Cabrera Urbán ${ }^{167}$, D. Caforio ${ }^{19 a, 19 b}$, O. Cakir ${ }^{3 a}$, P. Calafiura ${ }^{14}$, G. Calderini ${ }^{78}$, P. Calfayan $^{98}$, R. Calkins ${ }^{106}$, L.P. Caloba ${ }^{23 a}$, R. Caloi ${ }^{132 a, 132 b}$, D. Calvet ${ }^{33}$, S. Calvet ${ }^{33}$, R. Camacho Toro ${ }^{33}$, P. Camarri ${ }^{133 a, 133 b}$, D. Cameron ${ }^{117}$, L.M. Caminada ${ }^{14}$, S. Campana ${ }^{29}$, M. Campanelli ${ }^{77}$, V. Canale ${ }^{102 a, 102 b}$, F. Canelli ${ }^{30, g}$, A. Canepa ${ }^{159 a}$, J. Cantero ${ }^{80}$, R. Cantrill ${ }^{76}$, L. Capasso ${ }^{102 a, 102 b}$, M.D.M. Capeans Garrido ${ }^{29}$, I. Caprini ${ }^{25 a}$, M. Caprini ${ }^{25 a}$, D. Capriotti ${ }^{99}$, M. Capua ${ }^{36 a, 36 b}$, R. Caputo ${ }^{81}$, R. Cardarelli ${ }^{133 a}$, T. Carli ${ }^{29}$, G. Carlino ${ }^{102 a}$, L. Carminati ${ }^{89 a, 89 b}$, B. Caron ${ }^{85}$, S. Caron ${ }^{104}$, E. Carquin ${ }^{31 b}$, G.D. Carrillo Montoya ${ }^{173}$, A.A. $\operatorname{Carter}^{75}$, J.R. Carter ${ }^{27}$, J. Carvalho ${ }^{124 a, h}$, D. Casadei ${ }^{108}$, M.P. Casado ${ }^{11}$, M. Cascella ${ }^{122 a, 122 b}$, C. Caso 50a,50b,* , A.M. Castaneda Hernandez ${ }^{173, i}$, E. Castaneda-Miranda ${ }^{173}$, V. Castillo Gimenez ${ }^{167}$, N.F. Castro ${ }^{124 a}$, G. Cataldi ${ }^{72 a}$, P. Catastini ${ }^{57}$, A. Catinaccio ${ }^{29}$, J.R. Catmore ${ }^{29}$, A. Cattai ${ }^{29}$, G. Cattani ${ }^{133 a, 133 b}$, S. Caughron ${ }^{88}$, 
P. Cavalleri ${ }^{78}$, D. Cavalli ${ }^{89 a}$, M. Cavalli-Sforza ${ }^{11}$, V. Cavasinni ${ }^{122 a, 122 b}$, F. Ceradini $^{134 a, 134 b}$, A.S. Cerqueira ${ }^{23 b}$, A. Cerri ${ }^{29}$, L. Cerrito ${ }^{75}$, F. Cerutti ${ }^{47}$, S.A. Cetin ${ }^{18 b}$, A. Chafaq ${ }^{135 a}$, D. Chakraborty ${ }^{106}$, I. Chalupkova ${ }^{126}$, K. Chan ${ }^{2}$, B. Chapleau ${ }^{85}$, J.D. Chapman ${ }^{27}$, J.W. Chapman ${ }^{87}$, E. Chareyre ${ }^{78}$, D.G. Charlton ${ }^{17}$, V. Chavda ${ }^{82}$, C.A. Chavez Barajas ${ }^{29}$, S. Cheatham ${ }^{85}$, S. Chekanov ${ }^{5}$, S.V. Chekulaev ${ }^{159 a}$, G.A. Chelkov ${ }^{64}$, M.A. Chelstowska ${ }^{104}$, C. Chen ${ }^{63}$, H. Chen $^{24}$, S. Chen ${ }^{32 c}$, X. Chen ${ }^{173}$, A. Cheplakov ${ }^{64}$, R. Cherkaoui El Moursli ${ }^{135 e}$, V. Chernyatinn ${ }^{24}$, E. Cheu ${ }^{6}$, S.L. Cheung ${ }^{158}$, L. Chevalier ${ }^{136}$, G. Chiefari $^{102 a, 102 b}$, L. Chikovani ${ }^{51 a}$, J.T. Childers ${ }^{29}$, A. Chilingarov ${ }^{71}$, G. Chiodini ${ }^{72 a}$, A.S. Chisholm ${ }^{17}$, R.T. Chislett ${ }^{77}$, M.V. Chizhov ${ }^{64}$, G. Choudalakis ${ }^{30}$, S. Chouridou ${ }^{137}$, I.A. Christidii ${ }^{77}$, A. Christov ${ }^{48}$, D. Chromek-Burckhart ${ }^{29}$, M.L. Chu ${ }^{151}$, J. Chudoba ${ }^{125}$, G. Ciapetti ${ }^{132 a, 132 b}$, A.K. Ciftci ${ }^{3 a}$, R. Ciftci ${ }^{3 a}$, D. Cinca ${ }^{33}$, V. Cindro ${ }^{74}$, C. Ciocca ${ }^{19 a, 19 b}$, A. Ciocio ${ }^{14}$, M. Cirilli $^{87}$, P. Cirkovic ${ }^{12 b}$, M. Citterio ${ }^{89 a}$, M. Ciubancan ${ }^{25 a}$, A. Clark ${ }^{49}$, P.J. Clark ${ }^{45}$, W. Cleland ${ }^{123}$, J.C. Clemens ${ }^{83}$, B. Clement ${ }^{55}$, C. Clement ${ }^{146 a, 146 b}$, Y. Coadou ${ }^{83}$, M. Cobal ${ }^{164 a, 164 c}$, A. Coccaro ${ }^{138}$, J. Cochran ${ }^{63}$, J.G. Cogan ${ }^{143}$, J. Coggeshall ${ }^{165}$, E. Cogneras $^{178}$, J. Colas ${ }^{4}$, A.P. Colijn ${ }^{105}$, N.J. Collins ${ }^{17}$, C. Collins-Tooth ${ }^{53}$, J. Collot ${ }^{55}$, T. Colombo ${ }^{119 a, 119 b}$, G. Colon ${ }^{84}$, P. Conde Muiño $^{124 a}$, E. Coniavitis ${ }^{118}$, M.C. Conidi ${ }^{11}$, S.M. Consonni ${ }^{89 a, 89 b}$, V. Consorti ${ }^{48}$, S. Constantinescu ${ }^{25 a}$, C. Conta ${ }^{119 a, 119 b}$, G. Conti ${ }^{57}$, F. Conventi ${ }^{102 a, j}$, M. Cooke ${ }^{14}$, B.D. Cooper ${ }^{77}$, A.M. Cooper-Sarkar ${ }^{118}$, K. Copic ${ }^{14}$, T. Cornelissen ${ }^{175}$, M. Cor-

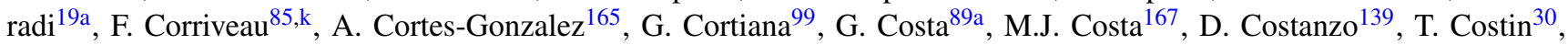
D. Côté ${ }^{29}$, L. Courneyea ${ }^{169}$, G. Cowan ${ }^{76}$, C. Cowden $^{27}$, B.E. Cox $^{82}$, K. Cranmer $^{108}$, F. Crescioli ${ }^{122 a, 122 b}$, M. Cristinziani ${ }^{20}$, G. Crosetti ${ }^{36 a, 36 b}$, R. Crupi ${ }^{72 a, 72 b}$, S. Crépé-Renaudin ${ }^{55}$, C.-M. Cuciucc ${ }^{25 a}$, C. Cuenca Almenar ${ }^{176}$, T. Cuhadar Donszelmann $^{139}$, M. Curatolo ${ }^{47}$, C.J. Curtis ${ }^{17}$, C. Cuthbert ${ }^{150}$, P. Cwetanski ${ }^{60}$, H. Czirr ${ }^{141}$, P. Czodrowski ${ }^{43}$, Z. Czyczula ${ }^{176}$, S. D’Auria ${ }^{53}$, M. D’Onofrio ${ }^{73}$, A. D’Orazio ${ }^{132 a, 132 b}$, M.J. Da Cunha Sargedas De Sousa ${ }^{124 a}$, C. Da Via ${ }^{82}$, W. Dabrowski ${ }^{37}$, A. Dafinca ${ }^{118}$, T. Dai ${ }^{87}$, C. Dallapiccola ${ }^{84}$, M. Dam ${ }^{35}$, M. Dameri ${ }^{50 a, 50 b}$, D.S. Damiani ${ }^{137}$, H.O. Danielsson ${ }^{29}$, V. Dao ${ }^{49}$, G. Darbo ${ }^{50 a}$, G.L. Darlea ${ }^{25 b}$, W. Davey ${ }^{20}$, T. Davidek ${ }^{126}$, N. Davidson ${ }^{86}$, R. Davidson ${ }^{71}$, E. Davies ${ }^{118, c}$, M. Davies ${ }^{93}$, A.R. Davison ${ }^{77}$, Y. Davygora ${ }^{58 a}$, E. Dawe ${ }^{142}$, I. Dawson ${ }^{139}$, R.K. Daya-Ishmukhametova ${ }^{22}, K^{2}$ De $^{7}$, R. de Asmundis ${ }^{102 a}$, S. De Castro ${ }^{19 a, 19 b}$, S. De Cecco ${ }^{78}$, J. de Graat ${ }^{98}$, N. De Groot ${ }^{104}$, P. de Jong ${ }^{105}$, C. De La Taille ${ }^{115}$, H. De la Torre ${ }^{80}$, F. De Lorenzi ${ }^{63}$, L. de Mora ${ }^{71}$, L. De Nooij ${ }^{105}$, D. De Pedis ${ }^{132 a}$, A. De Salvo ${ }^{132 a}$, U. De Sanctis ${ }^{164 a, 164 c}$, A. De Santo ${ }^{149}$, J.B. De Vivie De Regie ${ }^{115}$, G. De Zorzi ${ }^{132 a, 132 b}$, W.J. Dearnaley ${ }^{71}$, R. Debbe ${ }^{24}$, C. Debenedetti ${ }^{45}$, B. Dechenaux ${ }^{55}$, D.V. Dedovich $^{64}$, J. Degenhardt ${ }^{120}$, C. Del Papa ${ }^{164 a, 164 c}$, J. Del Peso ${ }^{80}$, T. Del Prete ${ }^{122 a, 122 b}$, T. Delemontex ${ }^{55}$, M. Deliyergiyev ${ }^{74}$, A. Dell'Acqua ${ }^{29}$, L. Dell'Asta ${ }^{21}$, M. Della Pietra ${ }^{102 a, j}$, D. della Volpe ${ }^{102 a, 102 b}$, M. Delmastro ${ }^{4}$, P.A. Delsart ${ }^{55}$, C. Deluca ${ }^{105}$, S. Demers ${ }^{176}$, M. Demichev ${ }^{64}$, B. Demirkoz ${ }^{11,1}$, J. Deng ${ }^{163}$, S.P. Denisov ${ }^{128}$, D. Derendarz ${ }^{38}$, J.E. Derkaoui ${ }^{135 d}$, F. Derue ${ }^{78}$, P. Dervan ${ }^{73}$, K. Desch ${ }^{20}$, E. Devetak ${ }^{148}$, P.O. Deviveiros ${ }^{105}$, A. Dewhurst ${ }^{129}$, B. DeWilde ${ }^{148}$, S. Dhaliwal ${ }^{158}$, R. Dhul$\operatorname{lipudi}^{24, m}$, A. Di Ciaccio ${ }^{133 a, 133 b}$, L. Di Ciaccio ${ }^{4}$, A. Di Girolamo ${ }^{29}$, B. Di Girolamo ${ }^{29}$, S. Di Luise ${ }^{134 a, 134 b}$, A. Di Mattia $^{173}$, B. Di Micco ${ }^{29}$, R. Di Nardo ${ }^{47}$, A. Di Simone ${ }^{133 a, 133 b}$, R. Di Sipio 19a,19b, M.A. Diaz ${ }^{31 a}$, E.B. Diehl ${ }^{87}$, J. Dietrich ${ }^{41}$, T.A. Dietzsch ${ }^{58 a}$, S. Diglio ${ }^{86}$, K. Dindar Yagci ${ }^{39}$, J. Dingfelder ${ }^{20}$, C. Dionisi ${ }^{132 a, 132 b}$, P. Dita ${ }^{25 a}$, S. Dita ${ }^{25 a}$, F. Dittus ${ }^{29}$, F. Djama ${ }^{83}$, T. Djobava ${ }^{51 b}$, M.A.B. do Vale ${ }^{23 c}$, A. Do Valle Wemans ${ }^{124 a, n}$, T.K.O. Doan ${ }^{4}$, M. Dobbs ${ }^{85}$, R. Dobinson ${ }^{29, *}$, D. Dobos ${ }^{29}$, E. Dobson ${ }^{29, \text { o }}$, J. Dodd ${ }^{34}$, C. Doglioni ${ }^{49}$, T. Doherty ${ }^{53}$, Y. Doi ${ }^{65, *}$, J. Dolejsi ${ }^{126}$, I. Dolenc ${ }^{74}$, Z. Dolezal ${ }^{126}$, B.A. Dolgoshein ${ }^{96, *}$, T. Dohmae ${ }^{155}$, M. Donadelli23d, M. Donega ${ }^{120}$, J. Donini ${ }^{33}$, J. Dopke ${ }^{29}$, A. Doria ${ }^{102 a}$, A. Dos Anjos $^{173}$, A. Dotti ${ }^{122 a, 122 b}$, M.T. Dova ${ }^{70}$, A.D. Doxiadis ${ }^{105}$, A.T. Doyle ${ }^{53}$, M. Dris ${ }^{9}$, J. Dubbert ${ }^{99}$, S. Dube ${ }^{14}$, E. Duchovni ${ }^{172}$, G. Duckeck $^{98}$, A. Dudarev ${ }^{29}$, F. Dudziak ${ }^{63}$, M. Dührssen ${ }^{29}$, I.P. Duerdoth ${ }^{82}$, L. Duflot ${ }^{115}$, M-A. Dufour ${ }^{85}$, M. Dunford $^{29}$, H. Duran Yildiz ${ }^{3 a}$, R. Duxfield ${ }^{139}$, M. Dwuznik ${ }^{37}$, F. Dydak ${ }^{29}$, M. Düren ${ }^{52}$, J. Ebke ${ }^{98}$, S. Eckweiler ${ }^{81}$, K. Edmonds $^{81}$, C.A. Edwards ${ }^{76}$, N.C. Edwards ${ }^{53}$, W. Ehrenfeld ${ }^{41}$, T. Eifert ${ }^{143}$, G. Eigen ${ }^{13}$, K. Einsweiler ${ }^{14}$, E. Eisenhandler ${ }^{75}$, T. Ekelof ${ }^{166}$, M. El Kacimi ${ }^{135 c}$, M. Ellert ${ }^{166}$, S. Elles $^{4}$, F. Ellinghaus ${ }^{81}$, K. Ellis ${ }^{75}$, N. Ellis ${ }^{29}$, J. Elmsheuser ${ }^{98}$, M. Elsing $^{29}$, D. Emeliyanov ${ }^{129}$, R. Engelmann ${ }^{148}$, A. Engl ${ }^{98}$, B. Epp ${ }^{61}$, A. Eppig ${ }^{87}$, J. Erdmann ${ }^{54}$, A. Ereditato ${ }^{16}$, D. Eriksson $^{146 a}$, J. Ernst ${ }^{1}$, M. Ernst ${ }^{24}$, J. Ernwein ${ }^{136}$, D. Errede ${ }^{165}$, S. Errede ${ }^{165}$, E. Ertel ${ }^{81}$, M. Escalier ${ }^{115}$, C. Escobar ${ }^{123}$, X. Espinal Curull ${ }^{11}$, B. Esposito ${ }^{47}$, F. Etienne ${ }^{83}$, A.I. Etienvre ${ }^{136}$, E. Etzion ${ }^{153}$, D. Evangelakou ${ }^{54}$, H. Evans ${ }^{60}$, L. Fabbri ${ }^{19 a, 19 b}$, C. Fabre $^{29}$, R.M. Fakhrutdinov ${ }^{128}$, S. Falciano ${ }^{132 a}$, Y. Fang ${ }^{173}$, M. Fanti ${ }^{89 a, 89 b}$, A. Farbin ${ }^{7}$, A. Farilla ${ }^{134 a}$, J. Farley ${ }^{148}$, T. Farooque ${ }^{158}$, S. Farrell ${ }^{163}$, S.M. Farrington ${ }^{118}$, P. Farthouat ${ }^{29}$, P. Fassnacht ${ }^{29}$, D. Fassouliotis ${ }^{8}$, B. Fatholahzadeh ${ }^{158}$,

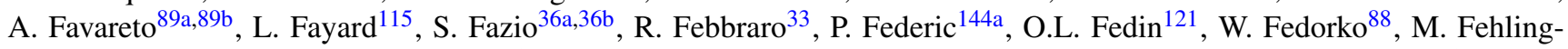
Kaschek $^{48}$, L. Feligioni ${ }^{83}$, D. Fellmann ${ }^{5}$, C. Feng ${ }^{32 d}$, E.J. Feng ${ }^{30}$, A.B. Fenyuk ${ }^{128}$, J. Ferencei ${ }^{144 b}$, W. Fernando ${ }^{5}$, S. Ferrag $^{53}$, J. Ferrando ${ }^{53}$, V. Ferrara ${ }^{41}$, A. Ferrari ${ }^{166}$, P. Ferrari ${ }^{105}$, R. Ferrari ${ }^{119 a}$, D.E. Ferreira de Lima ${ }^{53}$, A. Ferrer ${ }^{167}$, D. Ferrere $^{49}$, C. Ferretti ${ }^{87}$, A. Ferretto Parodi ${ }^{50,50 b}$, M. Fiascaris ${ }^{30}$, F. Fiedler ${ }^{81}$, A. Filipčič ${ }^{74}$, F. Filthaut ${ }^{104}$, M. Fincke-Keeler ${ }^{169}$, M.C.N. Fiolhais ${ }^{124 a, h}$, L. Fiorini ${ }^{167}$, A. Firan ${ }^{39}$, G. Fischer ${ }^{41}$, M.J. Fisher ${ }^{109}$, M. Flechl ${ }^{48}$, I. Fleck ${ }^{141}$, J. Fleckner ${ }^{81}$, P. Fleischmann $^{174}$, S. Fleischmann ${ }^{175}$, T. Flick ${ }^{175}$, A. Floderus ${ }^{79}$, L.R. Flores Castillo ${ }^{173}$, M.J. Flowerdew ${ }^{99}$, T. Fonseca Martin ${ }^{16}$, A. Formica ${ }^{136}$, A. Forti ${ }^{82}$, D. Fortin ${ }^{159 a}$, D. Fournier ${ }^{115}$, H. Fox ${ }^{71}$, P. Francavilla ${ }^{11}$, S. Franchino ${ }^{119 a, 119 b}$, D. Francis ${ }^{29}$, T. Frank ${ }^{172}$, S. Franz ${ }^{29}$, M. Fraternali ${ }^{119 a, 119 b}$, S. Fratina ${ }^{120}$, S.T. French ${ }^{27}$, C. Friedrich ${ }^{41}$, F. Friedrich ${ }^{43}$, R. Froeschl ${ }^{29}$, D. Froidevaux ${ }^{29}$, J.A. Frost ${ }^{27}$, C. Fukunaga ${ }^{156}$, E. Fullana Torregrosa ${ }^{29}$, B.G. Fulsom ${ }^{143}$, J. Fuster ${ }^{167}$, C. Gabaldon ${ }^{29}$, 
O. Gabizon ${ }^{172}$, T. Gadfort ${ }^{24}$, S. Gadomski ${ }^{49}$, G. Gagliardi ${ }^{50 a, 50 b}$, P. Gagnon ${ }^{60}$, C. Galea ${ }^{98}$, E.J. Gallas ${ }^{118}$, V. Gallo ${ }^{16}$, B.J. Gallop ${ }^{129}$, P. Gallus ${ }^{125}$, K.K. Gan ${ }^{109}$, Y.S. Gao ${ }^{143, \text { e }}$, A. Gaponenko ${ }^{14}$, F. Garberson ${ }^{176}$, M. Garcia-Sciveres ${ }^{14}$, C. García $^{167}$, J.E. García Navarro ${ }^{167}$, R.W. Gardner ${ }^{30}$, N. Garelli ${ }^{29}$, H. Garitaonandia ${ }^{105}$, V. Garonne ${ }^{29}$, J. Garvey ${ }^{17}$, C. Gatti ${ }^{47}$, G. Gaudio $^{119 a}$, B. Gaur ${ }^{141}$, L. Gauthier ${ }^{136}$, P. Gauzzi ${ }^{132 a, 132 b}$, I.L. Gavrilenko ${ }^{94}$, C. Gay ${ }^{168}$, G. Gaycken ${ }^{20}$, E.N. Gazis ${ }^{9}$, P. $\mathrm{Ge}^{32 \mathrm{~d}}$, Z. Gecse ${ }^{168}$, C.N.P. Gee ${ }^{129}$, D.A.A. Geerts ${ }^{105}$, Ch. Geich-Gimbel ${ }^{20}$, K. Gellerstedt ${ }^{146 a, 146 b}$, C. Gemme ${ }^{50 a}$, A. Gemmell $^{53}$, M.H. Genest ${ }^{55}$, S. Gentile ${ }^{132 a, 132 b}$, M. George ${ }^{54}$, S. George ${ }^{76}$, P. Gerlach ${ }^{175}$, A. Gershon ${ }^{153}$, C. Geweniger ${ }^{58 a}$, H. Ghazlane ${ }^{135 b}$, N. Ghodbane ${ }^{33}$, B. Giacobbe ${ }^{19 a}$, S. Giagu ${ }^{132 a, 132 b}$, V. Giakoumopoulou ${ }^{8}$, V. Giangiobbe ${ }^{11}$, F. Gianotti $^{29}$, B. Gibbard ${ }^{24}$, A. Gibson ${ }^{158}$, S.M. Gibson ${ }^{29}$, D. Gillberg ${ }^{28}$, A.R. Gillman ${ }^{129}$, D.M. Gingrich ${ }^{2, d}$, J. Ginzburg ${ }^{153}$, N. Giokaris ${ }^{8}$, M.P. Giordani ${ }^{164 c}$, R. Giordano ${ }^{102 a, 102 b}$, F.M. Giorgi ${ }^{15}$, P. Giovannini ${ }^{99}$, P.F. Giraud ${ }^{136}$, D. Giugni ${ }^{89 a}$, M. Giunta ${ }^{93}$, P. Giusti ${ }^{19 a}$, B.K. Gjelsten ${ }^{117}$, L.K. Gladilin ${ }^{97}$, C. Glasman ${ }^{80}$, J. Glatzer ${ }^{48}$, A. Glazov ${ }^{41}$, K.W. Glitza ${ }^{175}$, G.L. Glonti ${ }^{64}$, J.R. Goddard ${ }^{75}$, J. Godfrey ${ }^{142}$, J. Godlewski ${ }^{29}$, M. Goebel ${ }^{41}$, T. Göpfert ${ }^{43}$, C. Goeringer ${ }^{81}$, C. Gössling ${ }^{42}$, S. Goldfarb ${ }^{87}$, T. Golling ${ }^{176}$, A. Gomes ${ }^{124 a, b}$, L.S. Gomez Fajardo ${ }^{41}$, R. Gonçalo ${ }^{76}$, J. Goncalves Pinto Firmino Da Costa ${ }^{41}$, L. Gonella ${ }^{20}$, S. Gonzalez ${ }^{173}$, S. González de la Hoz ${ }^{167}$, G. Gonzalez Parra ${ }^{11}$, M.L. Gonzalez Silva ${ }^{26}$, S. GonzalezSevilla $^{49}$, J.J. Goodson ${ }^{148}$, L. Goossens ${ }^{29}$, P.A. Gorbounov ${ }^{95}$, H.A. Gordon ${ }^{24}$, I. Gorelov ${ }^{103}$, G. Gorfine ${ }^{175}$, B. Gorini ${ }^{29}$, E. Gorini ${ }^{72 a, 72 b}$, A. Gorišek ${ }^{74}$, E. Gornicki ${ }^{38}$, B. Gosdzik ${ }^{41}$, A.T. Goshaw ${ }^{5}$, M. Gosselink ${ }^{105}$, M.I. Gostkin ${ }^{64}$, I. Gough Eschrich $^{163}$, M. Gouighri ${ }^{135 a}$, D. Goujdami ${ }^{135 c}$, M.P. Goulette ${ }^{49}$, A.G. Goussiou ${ }^{138}$, C. Goy ${ }^{4}$, S. Gozpinar ${ }^{22}$, I. GrabowskaBold $^{37}$, P. Grafström ${ }^{29}$, K-J. Grahn ${ }^{41}$, F. Grancagnolo ${ }^{72 a}$, S. Grancagnolo ${ }^{15}$, V. Grassi ${ }^{148}$, V. Gratchev ${ }^{121}$, N. Grau ${ }^{34}$, H.M. Gray ${ }^{29}$, J.A. Gray ${ }^{148}$, E. Graziani ${ }^{134 a}$, O.G. Grebenyuk ${ }^{121}$, T. Greenshaw ${ }^{73}$, Z.D. Greenwood ${ }^{24, m}$, K. Gregersen ${ }^{35}$, I.M. Gregor ${ }^{41}$, P. Grenier ${ }^{143}$, J. Griffiths ${ }^{138}$, N. Grigalashvili ${ }^{64}$, A.A. Grillo ${ }^{137}$, S. Grinstein ${ }^{11}$, Y.V. Grishkevich ${ }^{97}$, J.-F. Grivaz $^{115}$, E. Gross ${ }^{172}$, J. Grosse-Knetter ${ }^{54}$, J. Groth-Jensen ${ }^{172}$, K. Grybel ${ }^{141}$, D. Guest ${ }^{176}$, C. Guicheney ${ }^{33}$, A. Guida ${ }^{72 a, 72 b}$, S. Guindon ${ }^{54}$, U. Gul ${ }^{53}$, H. Guler ${ }^{85, \text {, }}$, J. Gunther ${ }^{125}$, B. Guo ${ }^{158}$, J. Guo ${ }^{34}$, P. Gutierrez ${ }^{111}$, N. Guttman ${ }^{153}$, O. Gutzwiller ${ }^{173}$, C. $G_{u y o t}^{136}$, C. Gwenlan ${ }^{118}$, C.B. Gwilliam ${ }^{73}$, A. Haas ${ }^{143}$, S. Haas ${ }^{29}$, C. Haber ${ }^{14}$, H.K. Hadavand ${ }^{39}$, D.R. Hadley ${ }^{17}$, P. Haefner ${ }^{20}$, F. Hahn ${ }^{29}$, S. Haider ${ }^{29}$, Z. Hajduk ${ }^{38}$, H. Hakobyan ${ }^{177}$, D. Hall ${ }^{118}$, J. Haller ${ }^{54}$, K. Hamacher ${ }^{175}$, P. Hamal ${ }^{113}$, M. Hamer ${ }^{54}$, A. Hamilton ${ }^{145 b, q}$, S. Hamilton ${ }^{161}$, L. Han ${ }^{32 b}$, K. Hanagaki ${ }^{116}$, K. Hanawa ${ }^{160}$, M. Hance ${ }^{14}$, C. Handel ${ }^{81}$, P. Hanke ${ }^{58 a}$, J.R. Hansen ${ }^{35}$, J.B. Hansen ${ }^{35}$, J.D. Hansen ${ }^{35}$, P.H. Hansen ${ }^{35}$, P. Hansson ${ }^{143}$, K. Hara ${ }^{160}$, G.A. Hare ${ }^{137}$, T. Harenberg ${ }^{175}$, S. Harkusha ${ }^{90}$, D. Harper ${ }^{87}$, R.D. Harrington ${ }^{45}$, O.M. Harris ${ }^{138}$, K. Harrison ${ }^{17}$, J. Hartert ${ }^{48}$, F. Hartjes ${ }^{105}$, T. Haruyama ${ }^{65}$, A. Harvey ${ }^{56}$, S. Hasegawa ${ }^{101}$, Y. Hasegawa ${ }^{140}$, S. Hassani ${ }^{136}$, S. Haug ${ }^{16}$, M. Hauschild ${ }^{29}$, R. Hauser ${ }^{88}$, M. Havranek ${ }^{20}$, C.M. Hawkes ${ }^{17}$, R.J. Hawkings ${ }^{29}$, A.D. Hawkins ${ }^{79}$, D. Hawkins ${ }^{163}$, T. Hayakawa ${ }^{66}$, T. Hayashi ${ }^{160}$, D. Hay-

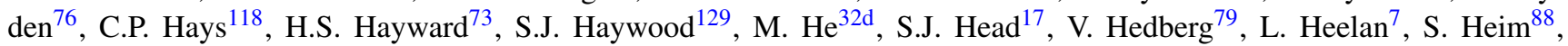
B. Heinemann ${ }^{14}$, S. Heisterkamp ${ }^{35}$, L. Helary ${ }^{4}$, C. Heller ${ }^{98}$, M. Heller ${ }^{29}$, S. Hellman ${ }^{146 a, 146 b}$, D. Hellmich ${ }^{20}$, C. Helsens ${ }^{11}$, R.C.W. Henderson ${ }^{71}$, M. Henke ${ }^{58 a}$, A. Henrichs ${ }^{54}$, A.M. Henriques Correia ${ }^{29}$, S. Henrot-Versille ${ }^{115}$, C. Hensel ${ }^{54}$, T. Henß ${ }^{175}$, C.M. Hernandez ${ }^{7}$, Y. Hernández Jiménez ${ }^{167}$, R. Herrberg ${ }^{15}$, G. Herten ${ }^{48}$, R. Hertenberger ${ }^{98}$, L. Hervas ${ }^{29}$, G.G. Hesketh ${ }^{77}$, N.P. Hessey ${ }^{105}$, E. Higón-Rodriguez ${ }^{167}$, J.C. Hill ${ }^{27}$, K.H. Hiller ${ }^{41}$, S. Hillert ${ }^{20}$, S.J. Hillier ${ }^{17}$, I. Hinchliffe ${ }^{14}$, E. Hines ${ }^{120}$, M. Hirose ${ }^{116}$, F. Hirsch ${ }^{42}$, D. Hirschbuehl ${ }^{175}$, J. Hobbs ${ }^{148}$, N. Hod ${ }^{153}$, M.C. Hodgkinson ${ }^{139}$, P. Hodgson ${ }^{139}$, A. Hoecker ${ }^{29}$, M.R. Hoeferkamp ${ }^{103}$, J. Hoffman ${ }^{39}$, D. Hoffmann ${ }^{83}$, M. Hohlfeld ${ }^{81}$, M. Holder ${ }^{141}$, S.O. Holmgren ${ }^{146 a}$, T. Holy ${ }^{127}$, J.L. Holzbauer ${ }^{88}$, T.M. Hong ${ }^{120}$, L. Hooft van Huysduynen ${ }^{108}$, C. Horn ${ }^{143}$, S. Horner ${ }^{48}$, J-Y. Hostachy ${ }^{55}$,

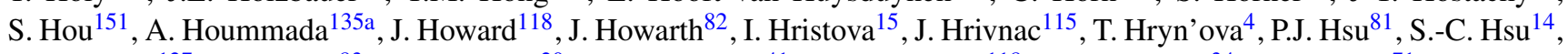
Z. Hubacek ${ }^{127}$, F. Hubaut ${ }^{83}$, F. Huegging ${ }^{20}$, A. Huettmann ${ }^{41}$, T.B. Huffman ${ }^{118}$, E.W. Hughes ${ }^{34}$, G. Hughes ${ }^{71}$, M. Huhti-

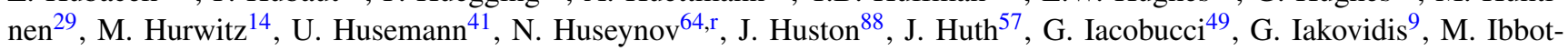
$\operatorname{son}^{82}$, I. Ibragimov ${ }^{141}$, L. Iconomidou-Fayard ${ }^{115}$, J. Idarraga ${ }^{115}$, P. Iengo ${ }^{102 a}$, O. Igonkina ${ }^{105}$, Y. Ikegami ${ }^{65}$, M. Ikeno ${ }^{65}$,

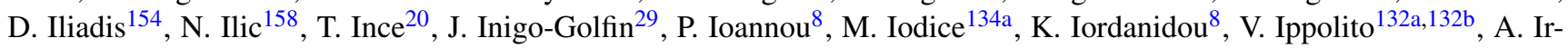
les Quiles ${ }^{167}$, C. Isaksson ${ }^{166}$, A. Ishikawa ${ }^{66}$, M. Ishino ${ }^{67}$, R. Ishmukhametov ${ }^{39}$, C. Issever $^{118}$, S. Istin ${ }^{18 a}$, A.V. Ivashin ${ }^{128}$, W. Iwanski ${ }^{38}$, H. Iwasaki ${ }^{65}$, J.M. Izen ${ }^{40}$, V. Izzo ${ }^{102 a}$, B. Jackson ${ }^{120}$, J.N. Jackson ${ }^{73}$, P. Jackson ${ }^{143}$, M.R. Jaekel ${ }^{29}$, V. Jain ${ }^{60}$, K. Jakobs ${ }^{48}$, S. Jakobsen ${ }^{35}$, T. Jakoubek ${ }^{125}$, J. Jakubek ${ }^{127}$, D.K. Jana ${ }^{111}$, E. Jansen ${ }^{77}$, H. Jansen ${ }^{29}$, A. Jantsch ${ }^{99}$, M. Janus ${ }^{48}$, G. Jarlskog ${ }^{79}$, L. Jeanty ${ }^{57}$, I. Jen-La Plante ${ }^{30}$, P. Jenni ${ }^{29}$, A. Jeremie ${ }^{4}$, P. Jež ${ }^{35}$, S. Jézéquel ${ }^{4}$, M.K. Jha ${ }^{19 a}$, H. Ji ${ }^{173}$, W. $\mathrm{Ji}^{81}$, J. Jia ${ }^{148}$, Y. Jiang ${ }^{32 b}$, M. Jimenez Belenguer ${ }^{41}$, S. Jin' ${ }^{32 \mathrm{a}}$, O. Jinnouchi ${ }^{157}$, M.D. Joergensen ${ }^{35}$, D. Joffe ${ }^{39}$, M. Johansen ${ }^{146 a, 146 b}$, K.E. Johansson ${ }^{146 a}$, P. Johansson ${ }^{139}$, S. Johnert ${ }^{41}$, K.A. Johns ${ }^{6}$, K. Jon-And ${ }^{146 a, 146 b}$, G. Jones ${ }^{170}$, R.W.L. Jones ${ }^{71}$, T.J. Jones ${ }^{73}$, C. Joram ${ }^{29}$, P.M. Jorge ${ }^{124 a}$, K.D. Joshi ${ }^{82}$, J. Jovicevic ${ }^{147}$, T. Jovin ${ }^{12 b}$, X. Ju ${ }^{173}$, C.A. Jung ${ }^{42}$, R.M. Jungst ${ }^{29}$, V. Juranek ${ }^{125}$, P. Jussel ${ }^{61}$, A. Juste Rozas ${ }^{11}$, S. Kabana ${ }^{16}$, M. Kaci ${ }^{167}$, A. Kaczmarska ${ }^{38}$, P. Kadlecik ${ }^{35}$, M. Kado ${ }^{115}$, H. Kagan ${ }^{109}$, M. Kagan ${ }^{57}$, E. Kajomovitz ${ }^{152}$, S. Kalinin ${ }^{175}$, L.V. Kalinovskaya ${ }^{64}$, S. Kama ${ }^{39}$, N. Kanaya ${ }^{155}$, M. Kaneda ${ }^{29}$, S. Kaneti ${ }^{27}$, T. Kanno ${ }^{157}$, V.A. Kantserov ${ }^{96}$, J. Kanzaki ${ }^{65}$, B. Kaplan ${ }^{176}$, A. Kapliy ${ }^{30}$, J. Kaplon ${ }^{29}$, D. Kar ${ }^{53}$, M. Karagounis ${ }^{20}$, K. Karakostas ${ }^{9}$, M. Karnevskiy ${ }^{41}$, V. Kartvelishvili ${ }^{71}$, A.N. Karyukhin ${ }^{128}$, L. Kashif ${ }^{173}$, G. Kasieczka ${ }^{58 b}$, R.D. Kass ${ }^{109}$, A. Kastanas ${ }^{13}$, M. Kataoka ${ }^{4}$, Y. Kataoka ${ }^{155}$, E. Katsoufis ${ }^{9}$, J. Katzy ${ }^{41}$, V. Kaushik ${ }^{6}$, K. Kawagoe ${ }^{69}$, 
T. Kawamoto ${ }^{155}$, G. Kawamura ${ }^{81}$, M.S. Kayl ${ }^{105}$, V.A. Kazanin ${ }^{107}$, M.Y. Kazarinov ${ }^{64}$, R. Keeler ${ }^{169}$, R. Kehoe ${ }^{39}$, M. Keil ${ }^{54}$, G.D. Kekelidze ${ }^{64}$, J.S. Keller ${ }^{138}$, M. Kenyon ${ }^{53}$, O. Kepka ${ }^{125}$, N. Kerschen ${ }^{29}$, B.P. Kerševan ${ }^{74}$, S. Kersten ${ }^{175}$, K. Kessoku ${ }^{155}$, J. Keung ${ }^{158}$, F. Khalil-zada ${ }^{10}$, H. Khandanyan ${ }^{165}$, A. Khanov ${ }^{112}$, D. Kharchenko ${ }^{64}$, A. Khodinov ${ }^{96}$, A. Khomich ${ }^{58 a}$, T.J. Khoo ${ }^{27}$, G. Khoriauli ${ }^{20}$, A. Khoroshilov ${ }^{175}$, V. Khovanskiy ${ }^{95}$, E. Khramov ${ }^{64}$, J. Khubua ${ }^{51 b}$, H. Kim ${ }^{146 a, 146 b}$, M.S. Kim ${ }^{2}$, S.H. Kim ${ }^{160}$, N. Kimura ${ }^{171}$, O. Kind ${ }^{15}$, B.T. King ${ }^{73}$, M. King ${ }^{66}$, R.S.B. King ${ }^{118}$, J. Kirk ${ }^{129}$, A.E. Kiryunin ${ }^{99}$, T. Kishimoto ${ }^{66}$, D. Kisielewska ${ }^{37}$, T. Kittelmann ${ }^{123}$, E. Kladiva ${ }^{144 b}$, M. Klein ${ }^{73}$, U. Klein ${ }^{73}$, K. Kleinknecht ${ }^{81}$, M. Klemetti ${ }^{85}$, A. Klier ${ }^{172}$, P. Klimek ${ }^{146 a, 146 b}$, A. Klimentov ${ }^{24}$, R. Klingenberg ${ }^{42}$, J.A. Klinger ${ }^{82}$, E.B. Klinkby ${ }^{35}$, T. Klioutchnikova ${ }^{29}$, P.F. Klok ${ }^{104}$, S. Klous ${ }^{105}$, E.-E. Kluge ${ }^{58 a}$, T. Kluge ${ }^{73}$, P. Kluit ${ }^{105}$, S. Kluth ${ }^{99}$, N.S. Knecht ${ }^{158}$, E. Kneringer ${ }^{61}$, E.B.F.G. Knoops ${ }^{83}$, A. Knue ${ }^{54}$, B.R. Ko ${ }^{44}$, T. Kobayashi ${ }^{155}$, M. Kobel ${ }^{43}$, M. Kocian ${ }^{143}$, P. Kodys ${ }^{126}$, K. Köneke ${ }^{29}$, A.C. König ${ }^{104}$, S. Koenig ${ }^{81}$, L. Köpke ${ }^{81}$, F. Koetsveld ${ }^{104}$, P. Koevesarki ${ }^{20}$, T. Koffas ${ }^{28}$, E. Koffeman ${ }^{105}$, L.A. Kogan ${ }^{118}$, S. Kohlmann ${ }^{175}$, F. Kohn ${ }^{54}$, Z. Kohout ${ }^{127}$, T. Kohriki ${ }^{65}$, T. Koi ${ }^{143}$, G.M. Kolachev ${ }^{107}$, H. Kolanoski ${ }^{15}$, V. Kolesnikov ${ }^{64}$, I. Koletsou ${ }^{89 a}$, J. Koll ${ }^{88}$, M. Kollefrath ${ }^{48}$, A.A. Komar ${ }^{94}$, Y. Komori ${ }^{155}$, T. Kondo ${ }^{65}$, T. Kono ${ }^{41, \mathrm{~s}}$, A.I. Kononov ${ }^{48}$, R. Konoplich ${ }^{108, t}$, N. Konstantinidis $^{77}$, S. Koperny ${ }^{37}$, K. Korcyl ${ }^{38}$, K. Kordas ${ }^{154}$, A. Korn ${ }^{118}$, A. Korol ${ }^{107}$, I. Korolkov ${ }^{11}$, E.V. Korolkova ${ }^{139}$, V.A. Korotkov $^{128}$, O. Kortner 99 , S. Kortner99, V.V. Kostyukhin ${ }^{20}$, S. Kotov ${ }^{99}$, V.M. Kotov ${ }^{64}$, A. Kotwal ${ }^{44}$, C. Kourkoumelis ${ }^{8}$, V. Kouskoura ${ }^{154}$, A. Koutsman ${ }^{159 a}$, R. Kowalewski ${ }^{169}$, T.Z. Kowalski ${ }^{37}$, W. Kozanecki ${ }^{136}$, A.S. Kozhin ${ }^{128}$, V. Kral ${ }^{127}$, V.A. Kramarenko ${ }^{97}$, G. Kramberger ${ }^{74}$, M.W. Krasny ${ }^{78}$, A. Krasznahorkay ${ }^{108}$, J. Kraus ${ }^{88}$, J.K. Kraus ${ }^{20}$, S. Kreiss ${ }^{108}$, F. Krejci $^{127}$, J. Kretzschmar ${ }^{73}$, N. Krieger ${ }^{54}$, P. Krieger ${ }^{158}$, K. Kroeninger ${ }^{54}$, H. Kroha ${ }^{99}$, J. Kroll ${ }^{120}$, J. Kroseberg ${ }^{20}$, J. Krstic ${ }^{12 a}$, U. Kruchonak ${ }^{64}$, H. Krüger ${ }^{20}$, T. Kruker ${ }^{16}$, N. Krumnack ${ }^{63}$, Z.V. Krumshteyn ${ }^{64}$, A. Kruth ${ }^{20}$, T. Kubota ${ }^{86}$, S. Kuday ${ }^{3 a}$, S. Kuehn ${ }^{48}$, A. Kugel ${ }^{58 c}$, T. Kuhl ${ }^{41}$, D. Kuhn ${ }^{61}$, V. Kukhtin ${ }^{64}$, Y. Kulchitsky ${ }^{90}$, S. Kuleshov ${ }^{31 b}$, C. Kummer ${ }^{98}$, M. Kuna ${ }^{78}$, J. Kunkle ${ }^{120}$, A. Kupco ${ }^{125}$, H. Kurashige ${ }^{66}$, M. Kurata ${ }^{160}$, Y.A. Kurochkin ${ }^{90}$, V. Kus ${ }^{125}$, E.S. Kuwertz ${ }^{147}$, M. Kuze ${ }^{157}$, J. Kvita ${ }^{142}$, R. Kwee ${ }^{15}$, A. La Rosa ${ }^{49}$, L. La Rotonda ${ }^{36 a, 36 b}$, L. Labarga ${ }^{80}$, J. Labbe ${ }^{4}$, S. Lablak ${ }^{135 a}$, C. Lacasta ${ }^{167}$, F. Lacava $^{132 a, 132 b}$, H. Lacker ${ }^{15}$, D. Lacour ${ }^{78}$, V.R. Lacuesta ${ }^{167}$, E. Ladygin ${ }^{64}$, R. Lafaye ${ }^{4}$, B. Laforge ${ }^{78}$, T. Lagouri ${ }^{80}$, S. Lai ${ }^{48}$, E. Laisne ${ }^{55}$, M. Lamanna ${ }^{29}$, L. Lambourne ${ }^{77}$, C.L. Lampen ${ }^{6}$, W. Lampl ${ }^{6}$, E. Lancon ${ }^{136}$, U. Landgraf ${ }^{48}$, M.P.J. Landon ${ }^{75}$, J.L. Lane ${ }^{82}$, C. Lange ${ }^{41}$, A.J. Lankford ${ }^{163}$, F. Lanni ${ }^{24}$, K. Lantzsch ${ }^{175}$, S. Laplace ${ }^{78}$, C. Lapoire $^{20}$, J.F. Laporte ${ }^{136}$, T. Lari ${ }^{89 a}$, A. Larner ${ }^{118}$, M. Lassnig ${ }^{29}$, P. Laurelli ${ }^{47}$, V. Lavorini ${ }^{36 a, 36 b}$, W. Lavrijsen ${ }^{14}$, P. Laycock ${ }^{73}$, O. Le Dortz ${ }^{78}$, E. Le Guirriec ${ }^{83}$, C. Le Maner ${ }^{158}$, E. Le Menedeu ${ }^{11}$, T. LeCompte ${ }^{5}$, F. Ledroit-Guillon ${ }^{55}$, H. Lee ${ }^{105}$, J.S.H. Lee ${ }^{116}$, S.C. Lee ${ }^{151}$, L. Lee ${ }^{176}$, M. Lefebvre ${ }^{169}$, M. Legendre ${ }^{136}$, B.C. LeGeyt ${ }^{120}$, F. Legger ${ }^{98}$, C. Leggett ${ }^{14}$, M. Lehmacher ${ }^{20}$, G. Lehmann Miotto ${ }^{29}$, X. Lei ${ }^{6}$, M.A.L. Leite ${ }^{23 d}$, R. Leitner ${ }^{126}$, D. Lellouch ${ }^{172}$, B. Lemmer ${ }^{54}$, V. Lendermann ${ }^{58 a}$, K.J.C. Leney ${ }^{145 b}$, T. Lenz ${ }^{105}$, G. Lenzen ${ }^{175}$, B. Lenzi ${ }^{29}$, K. Leonhardt ${ }^{43}$, S. Leontsinis ${ }^{9}$, F. Lepold ${ }^{58 a}$, C. Leroy ${ }^{93}$, J-R. Lessard ${ }^{169}$, C.G. Lester ${ }^{27}$, C.M. Lester ${ }^{120}$, J. Levêque ${ }^{4}$, D. Levin ${ }^{87}$, L.J. Levinson ${ }^{172}$, A. Lewis ${ }^{118}$, G.H. Lewis ${ }^{108}$, A.M. Leyko ${ }^{20}$, M. Leyton ${ }^{15}$, B. Li $^{83}$,

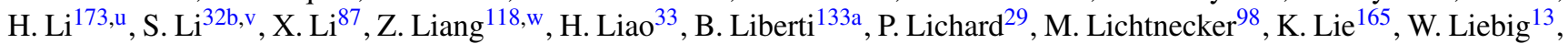

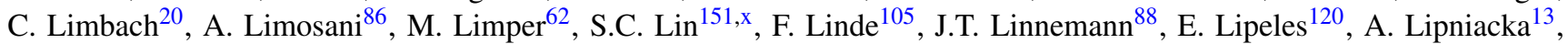

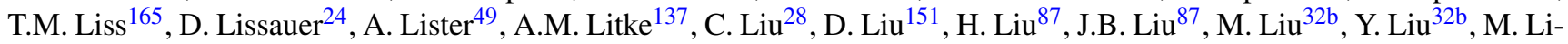
$\operatorname{van}^{119 a, 119 b}$, S.S.A. Livermore ${ }^{118}$, A. Lleres ${ }^{55}$, J. Llorente Merino ${ }^{80}$, S.L. Lloyd ${ }^{75}$, E. Lobodzinska ${ }^{41}$, P. Loch ${ }^{6}$, W.S. Lock$\operatorname{man}^{137}$, T. Loddenkoetter ${ }^{20}$, F.K. Loebinger ${ }^{82}$, A. Loginov ${ }^{176}$, C.W. Loh ${ }^{168}$, T. Lohse ${ }^{15}$, K. Lohwasser ${ }^{48}$, M. Lokajicek ${ }^{125}$, V.P. Lombardo ${ }^{4}$, R.E. Long ${ }^{71}$, L. Lopes ${ }^{124 a}$, D. Lopez Mateos ${ }^{57}$, J. Lorenz ${ }^{98}$, N. Lorenzo Martinez ${ }^{115}$, M. Losada ${ }^{162}$, P. Loscutoff ${ }^{14}$, F. Lo Sterzo ${ }^{132 a, 132 b}$, M.J. Losty ${ }^{159 a}$, X. Lou $^{40}$, A. Lounis ${ }^{115}$, K.F. Loureiro ${ }^{162}$, J. Love ${ }^{21}$, P.A. Love ${ }^{71}$, A.J. Lowe ${ }^{143, e}$, F. Lu ${ }^{32 a}$, H.J. Lubatti138, C. Luci132a,132b , A. Lucotte ${ }^{55}$, A. Ludwig ${ }^{43}$, D. Ludwig ${ }^{41}$, I. Ludwig ${ }^{48}$, J. Ludwig $^{48}$, F. Luehring ${ }^{60}$, G. Luijckx ${ }^{105}$, W. Lukas ${ }^{61}$, D. Lumb ${ }^{48}$, L. Luminari ${ }^{132 a}$, E. Lund ${ }^{117}$, B. Lund-Jensen ${ }^{147}$, B. Lundberg $^{79}$, J. Lundberg ${ }^{146 a, 146 b}$, J. Lundquist ${ }^{35}$, M. Lungwitz ${ }^{81}$, D. Lynn ${ }^{24}$, E. Lytken ${ }^{79}$, H. Ma ${ }^{24}$, L.L. Ma ${ }^{173}$, J.A. Macana Goia $^{93}$, G. Maccarrone ${ }^{47}$, A. Macchiolo ${ }^{99}$, B. Maček ${ }^{74}$, J. Machado Miguens ${ }^{124 a}$, R. Mackeprang ${ }^{35}$, R.J. Madaras ${ }^{14}$, W.F. Mader ${ }^{43}$, R. Maenner ${ }^{58 c}$, T. Maeno ${ }^{24}$, P. Mättig ${ }^{175}$, S. Mättig ${ }^{41}$, L. Magnoni ${ }^{29}$, E. Magradze ${ }^{54}$, K. Mahboubi ${ }^{48}$, S. Mahmoud ${ }^{73}$, G. Mahout ${ }^{17}$, C. Maiani ${ }^{136}$, C. Maidantchik ${ }^{23 a}$, A. Maio ${ }^{124 a, b}$, S. Majewskii ${ }^{24}$, Y. Makida $^{65}$, N. Makovec ${ }^{115}$, P. Mal ${ }^{136}$, B. Malaescu ${ }^{29}$, Pa. Malecki ${ }^{38}$, P. Malecki ${ }^{38}$, V.P. Maleev ${ }^{121}$, F. Malek ${ }^{55}$, U. Mallik ${ }^{62}$, D. Malon ${ }^{5}$, C. Malone $^{143}$, S. Maltezos ${ }^{9}$, V. Malyshev ${ }^{107}$, S. Malyukov ${ }^{29}$, R. Mameghani ${ }^{98}$, J. Mamuzic ${ }^{12 b}$, A. Manabe ${ }^{65}$, L. Mandelli ${ }^{89 a}$, I. Mandić ${ }^{74}$, R. Mandrysch ${ }^{15}$, J. Maneira ${ }^{124 a}$, P.S. Mangeard ${ }^{88}$, L. Manhaes de Andrade Filho ${ }^{23 a}$, A. Mann ${ }^{54}$, P.M. Manning $^{137}$, A. Manousakis-Katsikakis ${ }^{8}$, B. Mansoulie ${ }^{136}$, A. Mapelli ${ }^{29}$, L. Mapelli ${ }^{29}$, L. March ${ }^{80}$, J.F. Marchand ${ }^{28}$, F. Marchese $^{133 a, 133 b}$, G. Marchiori ${ }^{78}$, M. Marcisovsky ${ }^{125}$, C.P. Marino ${ }^{169}$, F. Marroquim ${ }^{23 a}$, Z. Marshall ${ }^{29}$, F.K. Martens ${ }^{158}$, S. Marti-

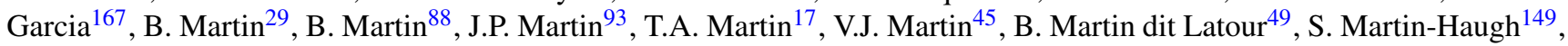
M. Martinez ${ }^{11}$, V. Martinez Outschoorn ${ }^{57}$, A.C. Martyniuk ${ }^{169}$, M. Marx ${ }^{82}$, F. Marzano ${ }^{132 a}$, A. Marzin ${ }^{111}$, L. Masetti ${ }^{81}$, T. Mashimo ${ }^{155}$, R. Mashinistov ${ }^{94}$, J. Masik ${ }^{82}$, A.L. Maslennikov ${ }^{107}$, I. Massa ${ }^{19 a, 19 b}$, G. Massaro ${ }^{105}$, N. Massol ${ }^{4}$, A. Mastroberardino $^{36 a, 36 b}$, T. Masubuchi ${ }^{155}$, P. Matricon ${ }^{115}$, H. Matsunaga ${ }^{155}$, T. Matsushita ${ }^{66}$, C. Mattravers ${ }^{118, \mathrm{c}}$, J. Maurer ${ }^{83}$, S.J. Maxfield ${ }^{73}$, A. Mayne ${ }^{139}$, R. Mazini ${ }^{151}$, M. Mazur ${ }^{20}$, L. Mazzaferro ${ }^{133 a, 133 b}$, M. Mazzanti ${ }^{89 a}$, S.P. Mc Kee ${ }^{87}$, A. Mc$\mathrm{Carn}^{165}$, R.L. McCarthy ${ }^{148}$, T.G. McCarthy ${ }^{28}$, N.A. McCubbin $^{129}$, K.W. McFarlane ${ }^{56}$, J.A. Mcfayden ${ }^{139}$, H. McGlone ${ }^{53}$, 
G. Mchedlidze ${ }^{51 b}$, T. Mclaughlan ${ }^{17}$, S.J. McMahon ${ }^{129}$, R.A. McPherson ${ }^{169, k}$, A. Meade ${ }^{84}$, J. Mechnich ${ }^{105}$, M. Mechtel ${ }^{175}$, M. Medinnis ${ }^{41}$, R. Meera-Lebbai ${ }^{111}$, T. Meguro ${ }^{116}$, R. Mehdiyev ${ }^{93}$, S. Mehlhase ${ }^{35}$, A. Mehta ${ }^{73}$, K. Meier ${ }^{58 a}$, B. Meirose ${ }^{79}$, C. Melachrinos ${ }^{30}$, B.R. Mellado Garcia ${ }^{173}$, F. Meloni ${ }^{89 a, 89 b}$, L. Mendoza Navas ${ }^{162}$, Z. Meng151,u A. Mengarelli19a,19b, S. Menke ${ }^{99}$, E. Meoni ${ }^{161}$, K.M. Mercurio ${ }^{57}$, P. Mermod ${ }^{49}$, L. Merola ${ }^{102 a, 102 b}$, C. Meroni ${ }^{89 a}$, F.S. Merritt ${ }^{30}$, H. Merritt ${ }^{109}$,

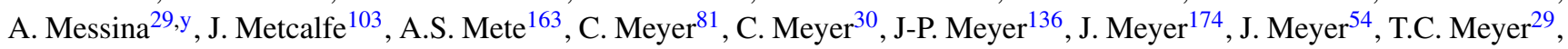
W.T. Meyer $^{63}$, J. Miao ${ }^{32 \mathrm{~d}}$, S. Michal ${ }^{29}$, L. Micu ${ }^{25 a}$, R.P. Middleton ${ }^{129}$, S. Migas ${ }^{73}$, L. Mijović ${ }^{41}$, G. Mikenberg ${ }^{172}$, M. Mikestikova ${ }^{125}$, M. Mikuž ${ }^{74}$, D.W. Miller ${ }^{30}$, R.J. Miller ${ }^{88}$, W.J. Mills ${ }^{168}$, C. Mills ${ }^{57}$, A. Milov ${ }^{172}$, D.A. Milstead ${ }^{146 a, 146 b}$, D. Milstein ${ }^{172}$, A.A. Minaenko ${ }^{128}$, M. Miñano Moya ${ }^{167}$, I.A. Minashvili ${ }^{64}$, A.I. Mincer ${ }^{108}$, B. Mindur ${ }^{37}$, M. Mineev ${ }^{64}$, Y. Ming ${ }^{173}$, L.M. Mir ${ }^{11}$, G. Mirabelli ${ }^{132 a}$, J. Mitrevski ${ }^{137}$, V.A. Mitsou ${ }^{167}$, S. Mitsui ${ }^{65}$, P.S. Miyagawa ${ }^{139}$, K. Miyazaki ${ }^{66}$, J.U. Mjörnmark ${ }^{79}$, T. Moa ${ }^{146 a, 146 b}$, V. Moeller ${ }^{27}$, K. Mönig ${ }^{41}$, N. Möser ${ }^{20}$, S. Mohapatra ${ }^{148}$, W. Mohr ${ }^{48}$, R. MolesValls $^{167}$, J. Molina-Perez ${ }^{29}$, J. Monk ${ }^{77}$, E. Monnier ${ }^{83}$, S. Montesano ${ }^{89 a, 89 b}$, F. Monticelli ${ }^{70}$, S. Monzani ${ }^{19 a, 19 b}$, R.W. Moore ${ }^{2}$, G.F. Moorhead ${ }^{86}$, C. Mora Herrera ${ }^{49}$, A. Moraes ${ }^{53}$, N. Morange ${ }^{136}$, J. Morel ${ }^{54}$, G. Morello ${ }^{36 a, 36 b}$, D. Moreno ${ }^{81}$, M. Moreno Llácer $^{167}$, P. Morettini ${ }^{50 a}$, M. Morgenstern ${ }^{43}$, M. Morii ${ }^{57}$, A.K. Morley ${ }^{29}$, G. Mornacchi ${ }^{29}$, J.D. Morris ${ }^{75}$, L. Morvaj ${ }^{101}$, H.G. Moser ${ }^{99}$, M. Mosidze ${ }^{51 b}$, J. Moss ${ }^{109}$, R. Mount ${ }^{143}$, E. Mountricha ${ }^{9, z}$, S.V. Mouraviev ${ }^{94}$, E.J.W. Moyse ${ }^{84}$, F. Mueller ${ }^{58 a}$, J. Mueller ${ }^{123}$, K. Mueller ${ }^{20}$, T.A. Müller ${ }^{98}$, T. Mueller ${ }^{81}$, D. Muenstermann ${ }^{29}$, Y. Munwes ${ }^{153}$, W.J. Murray ${ }^{129}$, I. Mussche ${ }^{105}$, E. Musto ${ }^{102 a, 102 b}$, A.G. Myagkov ${ }^{128}$, M. Myska ${ }^{125}$, J. Nadal ${ }^{11}$, K. Nagai ${ }^{160}$, K. Nagano ${ }^{65}$, A. Nagarkar ${ }^{109}$, Y. Nagasaka ${ }^{59}$, M. Nagel ${ }^{99}$, A.M. Nairz ${ }^{29}$, Y. Nakahama ${ }^{29}$, K. Nakamura ${ }^{155}$, T. Nakamura ${ }^{155}$, I. Nakano ${ }^{110}$, G. Nanava ${ }^{20}$, A. Napier ${ }^{161}$, R. Narayan ${ }^{58 b}$, M. Nash ${ }^{77, \mathrm{c}}$, T. Nattermann ${ }^{20}$, T. Naumann ${ }^{41}$, G. Navarro ${ }^{162}$, H.A. Neal ${ }^{87}$, P.Yu. Nechaeva ${ }^{94}$, T.J. Neep ${ }^{82}$, A. Negri ${ }^{119 a, 119 b}$, G. Negri ${ }^{29}$, S. Nektarijevic ${ }^{49}$, A. Nelson ${ }^{163}$, T.K. Nelson ${ }^{143}$, S. Nemecek ${ }^{125}$, P. Nemethy ${ }^{108}$, A.A. Nepomuceno $^{23 a}$, M. Nessi ${ }^{29}$,aa, M.S. Neubauer ${ }^{165}$, A. Neusiedl ${ }^{81}$, R.M. Neves ${ }^{108}$, P. Nevski ${ }^{24}$, P.R. Newman ${ }^{17}$, V. Nguyen Thi Hong $^{136}$, R.B. Nickerson ${ }^{118}$, R. Nicolaidou ${ }^{136}$, B. Nicquevert ${ }^{29}$, F. Niedercorn ${ }^{115}$, J. Nielsen ${ }^{137}$, N. Nikiforou ${ }^{34}$, A. Nikiforov $^{15}$, V. Nikolaenko ${ }^{128}$, I. Nikolic-Audit ${ }^{78}$, K. Nikolics ${ }^{49}$, K. Nikolopoulos ${ }^{24}$, H. Nilsen ${ }^{48}$, P. Nilsson ${ }^{7}$, Y. Ninomiya ${ }^{155}$, A. Nisati ${ }^{132 a}$, T. Nishiyama ${ }^{66}$, R. Nisius ${ }^{99}$, L. Nodulman ${ }^{5}$, M. Nomachi ${ }^{116}$, I. Nomidis ${ }^{154}$, M. Nordberg ${ }^{29}$, P.R. Norton ${ }^{129}$, J. Novakova ${ }^{126}$, M. Nozaki ${ }^{65}$, L. Nozka ${ }^{113}$, I.M. Nugent ${ }^{159}$, A.-E. Nuncio-Quiroz ${ }^{20}$, G. Nunes Hanninger ${ }^{86}$, T. Nunnemann ${ }^{98}$, E. Nurse $^{77}$, B.J. O’Brien ${ }^{45}$, S.W. O’Neale ${ }^{17, *}$, D.C. O'Neil ${ }^{142}$, V. O'Shea ${ }^{53}$, L.B. Oakes ${ }^{98}$, F.G. Oakham ${ }^{28, d}$,

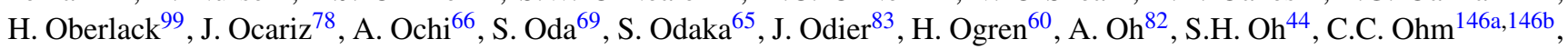
T. Ohshima ${ }^{101}$, S. Okada ${ }^{66}$, H. Okawa ${ }^{163}$, Y. Okumura ${ }^{101}$, T. Okuyama ${ }^{155}$, A. Olariu ${ }^{25 a}$, A.G. Olchevski ${ }^{64}$, S.A. Olivares Pino $^{31 a}$, M. Oliveira ${ }^{124 a, h}$, D. Oliveira Damazio ${ }^{24}$, E. Oliver Garcia ${ }^{167}$, D. Olivito ${ }^{120}$, A. Olszewski ${ }^{38}$, J. Olszowska ${ }^{38}$, A. Onofre ${ }^{124 a, a b}$, P.U.E. Onyisi ${ }^{30}$, C.J. Oram ${ }^{159 a}$, M.J. Oreglia ${ }^{30}$, Y. Oren ${ }^{153}$, D. Orestano ${ }^{134 a, 134 b}$, N. Orlando ${ }^{72 a, 72 b}$, I. Orlov ${ }^{107}$, C. Oropeza Barrera ${ }^{53}$, R.S. Orr ${ }^{158}$, B. Osculati ${ }^{50 a, 50 b}$, R. Ospanov ${ }^{120}$, C. Osuna ${ }^{11}$, G. Otero y Garzon ${ }^{26}$, J.P. Ottersbach $^{105}$, M. Ouchrif ${ }^{135 d}$, E.A. Ouellette ${ }^{169}$, F. Ould-Saada ${ }^{117}$, A. Ouraou ${ }^{136}$, Q. Ouyang ${ }^{32 a}$, A. Ovcharova ${ }^{14}$, M. Owen $^{82}$, S. Owen ${ }^{139}$, V.E. Ozcan ${ }^{18 a}$, N. Ozturk ${ }^{7}$, A. Pacheco Pages ${ }^{11}$, C. Padilla Aranda ${ }^{11}$, S. Pagan Griso ${ }^{14}$, E. Paganis ${ }^{139}$, F. Paige ${ }^{24}$, P. Pais $^{84}$, K. Pajchel ${ }^{117}$, G. Palacino ${ }^{159 b}$, C.P. Paleari ${ }^{6}$, S. Palestini ${ }^{29}$, D. Pallin ${ }^{33}$, A. Palma ${ }^{124 a}$, J.D. Palmer ${ }^{17}$, Y.B. Pan ${ }^{173}$, E. Panagiotopoulou ${ }^{9}$, P. Pani ${ }^{105}$, N. Panikashvili ${ }^{87}$, S. Panitkin ${ }^{24}$, D. Pantea ${ }^{25 a}$, A. Papadelis ${ }^{146 a}$, Th.D. Papadopoulou ${ }^{9}$, A. Paramonov , D. Paredes Hernandez ${ }^{33}$, W. Park ${ }^{24, a c}$, M.A. Parker ${ }^{27}$, F. Parodi ${ }^{50,50 b}$, J.A. Parsons ${ }^{34}$, U. Parzefall ${ }^{48}$, S. Pashapour ${ }^{54}$, E. Pasqualucci ${ }^{132 a}$, S. Passaggio ${ }^{50 a}$, A. Passeri ${ }^{134 a}$, F. Pastore ${ }^{134 a, 134 b}$, Fr. Pastore ${ }^{76}$, G. Pásztor ${ }^{49, a d}$, S. Pataraia ${ }^{175}$, N. Patel ${ }^{150}$, J.R. Pater ${ }^{82}$, S. Patricelli ${ }^{102 a, 102 b}$, T. Pauly ${ }^{29}$, M. Pecsy ${ }^{144 a}$, M.I. Pedraza Morales ${ }^{173}$, S.V. Peleganchuk $^{107}$, D. Pelikan ${ }^{166}$, H. Peng ${ }^{32 b}$, B. Penning ${ }^{30}$, A. Penson ${ }^{34}$, J. Penwell ${ }^{60}$, M. Perantoni ${ }^{23 a}$, K. Perez $^{34, a e}$, T. Perez Cavalcanti $^{41}$, E. Perez Codina ${ }^{159 a}$, M.T. Pérez García-Estañ ${ }^{167}$, V. Perez Reale ${ }^{34}$, L. Perini ${ }^{89 a, 89 b}$, H. Pernegger ${ }^{29}$, R. Perrino $^{72 a}$, P. Perrodo ${ }^{4}$, S. Persembe ${ }^{3 a}$, V.D. Peshekhonov ${ }^{64}$, K. Peters ${ }^{29}$, B.A. Petersen ${ }^{29}$, J. Petersen ${ }^{29}$, T.C. Petersen ${ }^{35}$, E. Petit $^{4}$, A. Petridis ${ }^{154}$, C. Petridou ${ }^{154}$, E. Petrolo ${ }^{132 a}$, F. Petrucci ${ }^{134 a, 134 b}$, D. Petschull ${ }^{41}$, M. Petteni ${ }^{142}$, R. Pezoa ${ }^{31 b}$, A. Phan $^{86}$, P.W. Phillips ${ }^{129}$, G. Piacquadio ${ }^{29}$, A. Picazio ${ }^{49}$, E. Piccaro ${ }^{75}$, M. Piccinini ${ }^{19 a, 19 b}$, S.M. Piec ${ }^{41}$, R. Piegaia ${ }^{26}$, D.T. Pignotti $^{109}$, J.E. Pilcher ${ }^{30}$, A.D. Pilkington ${ }^{82}$, J. Pina ${ }^{124 a, b}$, M. Pinamonti ${ }^{164 a, 164 c}$, A. Pinder ${ }^{118}$, J.L. Pinfold ${ }^{2}$, B. Pinto ${ }^{124 a}$, C. Pizio ${ }^{89 a, 89 b}$, M. Plamondon ${ }^{169}$, M.-A. Pleier ${ }^{24}$, E. Plotnikova ${ }^{64}$, A. Poblaguev ${ }^{24}$, S. Poddar ${ }^{58 a}$, F. Podlyski ${ }^{33}$, L. Poggioli $^{115}$, T. Poghosyan ${ }^{20}$, M. Pohl ${ }^{49}$, F. Polci ${ }^{55}$, G. Polesello ${ }^{119 a}$, A. Policicchio ${ }^{36 a, 36 b}$, A. Polini ${ }^{19 a}$, J. Poll ${ }^{75}$, V. Polychron$\operatorname{akos}^{24}$, D. Pomeroy ${ }^{22}$, K. Pommès ${ }^{29}$, L. Pontecorvo ${ }^{132 a}$, B.G. Pope ${ }^{88}$, G.A. Popeneciu ${ }^{25 a}$, D.S. Popovic ${ }^{12 a}$, A. Poppleton ${ }^{29}$, X. Portell Bueso ${ }^{29}$, G.E. Pospelov ${ }^{99}$, S. Pospisil ${ }^{127}$, I.N. Potrap ${ }^{99}$, C.J. Potter ${ }^{149}$, C.T. Potter ${ }^{14}$, G. Poulard ${ }^{29}$, J. Poveda ${ }^{60}$, V. Pozdnyakov ${ }^{64}$, R. Prabhu ${ }^{77}$, P. Pralavorio ${ }^{83}$, A. Pranko ${ }^{14}$, S. Prasad ${ }^{29}$, R. Pravahan ${ }^{24}$, S. Prell ${ }^{63}$, K. Pretzl ${ }^{16}$, D. Price ${ }^{60}$, J. Price ${ }^{73}$, L.E. Price ${ }^{5}$, D. Prieur ${ }^{123}$, M. Primavera ${ }^{72 a}$, K. Prokofiev ${ }^{108}$, F. Prokoshin ${ }^{31 b}$, S. Protopopescu ${ }^{24}$, J. Proudfoot ${ }^{5}$, X. Prudent ${ }^{43}$, M. Przybycien ${ }^{37}$, H. Przysiezniak ${ }^{4}$, S. Psoroulas ${ }^{20}$, E. Ptacek $^{114}$, E. Pueschel ${ }^{84}$, J. Purdham ${ }^{87}$, M. Purohit $^{24, a c}$, P. Puzo ${ }^{115}$, Y. Pylypchenko ${ }^{62}$, J. Qian ${ }^{87}$, A. Quadt ${ }^{54}$, D.R. Quarrie ${ }^{14}$, W.B. Quayle ${ }^{173}$, F. Quinonez ${ }^{31 a}$, M. Raas $^{104}$, V. Radescu ${ }^{41}$, P. Radloff ${ }^{14}$, T. Rador ${ }^{18 a}$, F. Ragusa ${ }^{89 a, 89 b}$, G. Rahal ${ }^{178}$, A.M. Rahimi ${ }^{109}$, D. Rahm ${ }^{24}$, S. Rajagopalan ${ }^{24}$, M. Rammensee ${ }^{48}$, M. Rammes ${ }^{141}$, A.S. Randle-Conde ${ }^{39}$, K. Randrianarivony ${ }^{28}$, F. Rauscher ${ }^{98}$, T.C. Rave ${ }^{48}$, M. Raymond ${ }^{29}$, 
A.L. $\operatorname{Read}^{117}$, D.M. Rebuzzi ${ }^{119 a, 119 b}$, A. Redelbach ${ }^{174}$, G. Redlinger $^{24}$, R. Reece ${ }^{120}$, K. Reeves ${ }^{40}$, E. Reinherz-Aronis ${ }^{153}$, A. Reinsch ${ }^{114}$, I. Reisinger ${ }^{42}$, C. Rembser ${ }^{29}$, Z.L. Ren ${ }^{151}$, A. Renaud ${ }^{115}$, M. Rescigno ${ }^{132 a}$, S. Resconi ${ }^{89 a}$, B. Resende ${ }^{136}$, P. Reznicek ${ }^{98}$, R. Rezvani ${ }^{158}$, R. Richter ${ }^{99}$, E. Richter-Was ${ }^{4, \text { af }}$, M. Ridel ${ }^{78}$, M. Rijpstra ${ }^{105}$, M. Rijssenbeek ${ }^{148}$, A. Rimoldi $^{119 a, 119 b}$, L. Rinaldi ${ }^{19 a}$, R.R. Rios ${ }^{39}$, I. Riu ${ }^{11}$, G. Rivoltella ${ }^{89 a, 89 b}$, F. Rizatdinova ${ }^{112}$, E. Rizvi ${ }^{75}$, S.H. Robertson ${ }^{85, k}$, A. Robichaud-Veronneau ${ }^{118}$, D. Robinson ${ }^{27}$, J.E.M. Robinson ${ }^{77}$, A. Robson ${ }^{53}$, J.G. Rocha de Lima ${ }^{106}$, C. Roda ${ }^{122 a, 122 b}$, D. Roda Dos Santos ${ }^{29}$, A. Roe ${ }^{54}$, S. Roe ${ }^{29}$, O. Røhne ${ }^{117}$, S. Rolli ${ }^{161}$, A. Romaniouk ${ }^{96}$, M. Romano ${ }^{19 a, 19 b}$, G. Romeo ${ }^{26}$, E. Romero Adam ${ }^{167}$, L. Roos ${ }^{78}$, E. Ros $^{167}$, S. Rosati ${ }^{132 a}$, K. Rosbach ${ }^{49}$, A. Rose ${ }^{149}$, M. Rose ${ }^{76}$, G.A. Rosenbaum ${ }^{158}$, E.I. Rosenberg ${ }^{63}$, P.L. Rosendahl ${ }^{13}$, O. Rosenthal ${ }^{141}$, L. Rosselet ${ }^{49}$, V. Rossetti ${ }^{11}$, E. Rossi ${ }^{132 a, 132 b}$, L.P. Rossi ${ }^{50 a}$, M. Ro$\operatorname{taru}^{25 a}$, I. Roth ${ }^{172}$, J. Rothberg ${ }^{138}$, D. Rousseau ${ }^{115}$, C.R. Royon ${ }^{136}$, A. Rozanov ${ }^{83}$, Y. Rozen ${ }^{152}$, X. Ruan ${ }^{32 a, a g}$, F. Rubbo ${ }^{11}$, I. Rubinskiy ${ }^{41}$, B. Ruckert ${ }^{98}$, N. Ruckstuhl ${ }^{105}$, V.I. Rud ${ }^{97}$, C. Rudolph ${ }^{43}$, G. Rudolph ${ }^{61}$, F. Rühr ${ }^{6}$, F. Ruggieri ${ }^{134 a, 134 b}$, A. Ruiz-Martinez ${ }^{63}$, L. Rumyantsev ${ }^{64}$, Z. Rurikova ${ }^{48}$, N.A. Rusakovich ${ }^{64}$, J.P. Rutherfoord ${ }^{6}$, C. Ruwiedel ${ }^{14}$, P. Ruzicka $^{125}$, Y.F. Ryabov ${ }^{121}$, P. Ryan ${ }^{88}$, M. Rybar ${ }^{126}$, G. Rybkin ${ }^{115}$, N.C. Ryder ${ }^{118}$, A.F. Saavedra ${ }^{150}$, I. Sadeh ${ }^{153}$, H.FW. Sadrozinski ${ }^{137}$, R. Sadykov ${ }^{64}$, F. Safai Tehrani ${ }^{132 a}$, H. Sakamoto ${ }^{155}$, G. Salamanna ${ }^{75}$, A. Salamon ${ }^{133 a}$, M. Saleem ${ }^{111}$, D. Salek $^{29}$, D. Salihagic ${ }^{99}$, A. Salnikov ${ }^{143}$, J. Salt ${ }^{167}$, B.M. Salvachua Ferrando ${ }^{5}$, D. Salvatore ${ }^{36 a, 36 b}$, F. Salvatore ${ }^{149}$, A. Salvucci ${ }^{104}$, A. Salzburger ${ }^{29}$, D. Sampsonidis ${ }^{154}$, B.H. Samset ${ }^{117}$, A. Sanchez ${ }^{102 a, 102 b}$, V. Sanchez Martinez ${ }^{167}$, H. Sandaker $^{13}$, H.G. Sander ${ }^{81}$, M.P. Sanders ${ }^{98}$, M. Sandhoff ${ }^{175}$, T. Sandoval $^{27}$, C. Sandoval ${ }^{162}$, R. Sandstroem ${ }^{99}$, D.P.C. Sankey ${ }^{129}$, A. Sansoni ${ }^{47}$, C. Santamarina Rios ${ }^{85}$, C. Santoni ${ }^{33}$, R. Santonico ${ }^{133 a, 133 b}$, H. Santos ${ }^{124 a}$, J.G. Saraiva ${ }^{124 a}$, T. Sarangi ${ }^{173}$, E. Sarkisyan-Grinbaum ${ }^{7}$, F. Sarri ${ }^{122 a, 122 b}$, G. Sartisohn ${ }^{175}$, O. Sasaki ${ }^{65}$, N. Sasao ${ }^{67}$, I. Satsounkevitch ${ }^{90}$, G. Sauvage ${ }^{4}$, E. $S_{a u v a n}^{4}$, J.B. Sauvan ${ }^{115}$, P. Savard ${ }^{158, d}$, V. Savinov ${ }^{123}$, D.O. Savu ${ }^{29}$, L. Sawyer ${ }^{24, m}$, D.H. Saxon ${ }^{53}$, J. Saxon ${ }^{120}$, C. Sbarra ${ }^{19 a}$, A. Sbrizzi ${ }^{19 a}{ }^{19 b}$, O. Scallon ${ }^{93}$, D.A. Scannicchio ${ }^{163}$, M. Scarcella ${ }^{150}$, J. Schaarschmidt ${ }^{115}$, P. Schacht ${ }^{99}$, D. Schaefer ${ }^{120}$, U. Schäfer ${ }^{81}$, S. Schaepe ${ }^{20}$, S. Schaetzel ${ }^{58 b}$, A.C. Schaffer ${ }^{115}$, D. Schaile ${ }^{98}$, R.D. Schamberger ${ }^{148}$, A.G. Schamov ${ }^{107}$, V. Scharf ${ }^{58 a}$, V.A. Schegelsky ${ }^{121}$, D. Scheirich ${ }^{87}$, M. Schernau ${ }^{163}$, M.I. Scherzer ${ }^{34}$, C. Schiavi ${ }^{50 a, 50 b}$, J. Schieck ${ }^{98}$, M. Schioppa ${ }^{36 a, 36 b}$, S. Schlenker ${ }^{29}$, E. Schmidt ${ }^{48}$, K. Schmieden ${ }^{20}$, C. Schmitt ${ }^{81}$, S. Schmitt ${ }^{58 b}$, M. Schmitz ${ }^{20}$, B. Schneider ${ }^{16}$, U. Schnoor ${ }^{43}$, A. Schöning ${ }^{58 b}$, A.L.S. Schorlemmer ${ }^{54}$, M. Schott ${ }^{29}$, D. Schouten ${ }^{159 a}$, J. Schovancova ${ }^{125}$, M. Schram ${ }^{85}$, C. Schroeder ${ }^{81}$, N. Schroer ${ }^{58 c}$, M.J. Schultens ${ }^{20}$, J. Schultes ${ }^{175}$, H.-C. Schultz-Coulon ${ }^{58 a}$, H. Schulz ${ }^{15}$, J.W. Schumacher ${ }^{20}$, M. Schumacher ${ }^{48}$, B.A. Schumm ${ }^{137}$, Ph. Schune ${ }^{136}$, C. Schwanenberger ${ }^{82}$, A. Schwartzman ${ }^{143}$, Ph. Schwemling ${ }^{78}$, R. Schwienhorst ${ }^{88}$, R. Schwierz ${ }^{43}$, J. Schwindling ${ }^{136}$, T. Schwindt ${ }^{20}$, M. Schwoerer ${ }^{4}$, G. Sciolla ${ }^{22}$, W.G. Scott ${ }^{129}$, J. Searcy ${ }^{114}$, G. Sedov ${ }^{41}$, E. Sedykh ${ }^{121}$, S.C. Seidel ${ }^{103}$, A. Seiden ${ }^{137}$, F. Seifert ${ }^{43}$, J.M. Seixas ${ }^{23 a}$, G. Sekhniaidze $^{102 a}$, S.J. Sekula ${ }^{39}$, K.E. Selbach ${ }^{45}$, D.M. Seliverstov ${ }^{121}$, B. Sellden ${ }^{146 a}$, G. Sellers ${ }^{73}$, M. Seman ${ }^{144 b}$, N. SempriniCesari $^{19 a, 19 b}$, C. Serfon ${ }^{98}$, L. Serin ${ }^{115}$, L. Serkin ${ }^{54}$, R. Seuster ${ }^{99}$, H. Severini ${ }^{111}$, A. Sfyrla ${ }^{29}$, E. Shabalina ${ }^{54}$, M. Shamim ${ }^{114}$, L.Y. Shan ${ }^{32 a}$, J.T. Shank ${ }^{21}$, Q.T. Shao ${ }^{86}$, M. Shapiro ${ }^{14}$, P.B. Shatalov ${ }^{95}$, K. Shaw ${ }^{164 a, 164 c}$, D. Sherman ${ }^{176}$, P. Sherwood ${ }^{77}$, A. Shibata ${ }^{108}$, H. Shichi ${ }^{101}$, S. Shimizu ${ }^{29}$, M. Shimojima ${ }^{100}$, T. Shin ${ }^{56}$, M. Shiyakova ${ }^{64}$, A. Shmeleva ${ }^{94}$, M.J. Shochet ${ }^{30}$, D. Short ${ }^{118}$, S. Shrestha ${ }^{63}$, E. Shulga ${ }^{96}$, M.A. Shupe ${ }^{6}$, P. Sicho ${ }^{125}$, A. Sidoti ${ }^{132 a}$, F. Siegert ${ }^{48}$, Dj. Sijacki ${ }^{12 a}$, O. Silbert ${ }^{172}$, J. Silva ${ }^{124 a}$, Y. Silver ${ }^{153}$, D. Silverstein ${ }^{143}$, S.B. Silverstein ${ }^{146 a}$, V. Simak ${ }^{127}$, O. Simard ${ }^{136}$, Lj. Simic ${ }^{12 a}$, S. Simion ${ }^{115}$, E. Simioni ${ }^{81}$, B. Simmons ${ }^{77}$, R. Simoniello ${ }^{89 a, 89 b}$, M. Simonyan ${ }^{35}$, P. Sinervo ${ }^{158}$, N.B. Sinev ${ }^{114}$, V. Sipica ${ }^{141}$, G. Siragusa ${ }^{174}$, A. $\operatorname{Sircar}^{24}$, A.N. Sisakyan ${ }^{64}$, S.Yu. Sivoklokov ${ }^{97}$, J. Sjölin ${ }^{146 a, 146 b}$, T.B. Sjursen ${ }^{13}$, L.A. Skinnari ${ }^{14}$, H.P. Skottowe ${ }^{57}$, K. Skovpen ${ }^{107}$, P. Skubic ${ }^{111}$, M. Slater ${ }^{17}$, T. Slavicek ${ }^{127}$, K. Sliwa ${ }^{161}$, V. Smakhtin ${ }^{172}$, B.H. Smart ${ }^{45}$, S.Yu. Smirnov ${ }^{96}$, Y. Smirnov ${ }^{96}$, L.N. Smirnova ${ }^{97}$, O. Smirnova ${ }^{79}$, B.C. Smith ${ }^{57}$, D. Smith ${ }^{143}$, K.M. Smith ${ }^{53}$, M. Smizanska ${ }^{71}$, K. Smolek ${ }^{127}$, A.A. Snesarev ${ }^{94}$, S.W. Snow ${ }^{82}$, J. Snow ${ }^{111}$, S. Snyder ${ }^{24}$, R. Sobie ${ }^{169, k}$, J. Sodomka ${ }^{127}$, A. Soffer ${ }^{153}$, C.A. Solans ${ }^{167}$, M. So$\operatorname{lar}^{127}$, J. Solc ${ }^{127}$, E.Yu. Soldatov ${ }^{96}$, U. Soldevila ${ }^{167}$, E. Solfaroli Camillocci ${ }^{132 a, 132 b}$, A.A. Solodkov ${ }^{128}$, O.V. Solovyanov ${ }^{128}$, N. Soni ${ }^{2}$, V. Sopko ${ }^{127}$, B. Sopko ${ }^{127}$, M. Sosebee ${ }^{7}$, R. Soualah ${ }^{164 a, 164 c}$, A. Soukharev ${ }^{107}$, S. Spagnolo ${ }^{72 a, 72 b}$, F. Spanò ${ }^{76}$, R. Spighi ${ }^{19 a}$, G. Spigo $^{29}$, F. Spila ${ }^{132 a, 132 b}$, R. Spiwoks ${ }^{29}$, M. Spousta ${ }^{126}$, T. Spreitzer ${ }^{158}$, B. Spurlock ${ }^{7}$, R.D. St. Denis $^{53}$, J. Stahlman ${ }^{120}$, R. Stamen ${ }^{58 a}$, E. Stanecka ${ }^{38}$, R.W. Stanek ${ }^{5}$, C. Stanescu ${ }^{134 a}$, M. Stanescu-Bellu ${ }^{41}$, S. Stapnes ${ }^{117}$, E.A. Starchenko ${ }^{128}$, J. Stark ${ }^{55}$, P. Staroba ${ }^{125}$, P. Starovoitov ${ }^{41}$, A. Staude ${ }^{98}$, P. Stavina ${ }^{144 a}$, G. Steele ${ }^{53}$, P. Steinbach ${ }^{43}$, P. Steinberg ${ }^{24}$, I. Stekl ${ }^{127}$, B. Stelzer ${ }^{142}$, H.J. Stelzer ${ }^{88}$, O. Stelzer-Chilton ${ }^{159 a}$, H. Stenzel ${ }^{52}$, S. Stern ${ }^{99}$, G.A. Stewart ${ }^{29}$, J.A. Stillings ${ }^{20}$, M.C. Stockton ${ }^{85}$, K. Stoerig ${ }^{48}$, G. Stoicea ${ }^{25 a}$, S. Stonjek ${ }^{99}$, P. Strachota ${ }^{126}$, A.R. Stradling 7 , A. Straessner $^{43}$, J. Strandberg ${ }^{147}$, S. Strandberg ${ }^{146 a, 146 b}$, A. Strandlie ${ }^{117}$, M. Strang ${ }^{109}$, E. Strauss ${ }^{143}$, M. Strauss ${ }^{111}$, P. Strizenec ${ }^{144 b}$, R. Ströhmer ${ }^{174}$, D.M. Strom ${ }^{114}$, J.A. Strong ${ }^{76, *}$, R. Stroynowski ${ }^{39}$, J. Strube ${ }^{129}$, B. Stugu ${ }^{13}$, I. Stumer ${ }^{24, *}$, J. Stupak ${ }^{148}$, P. Sturm ${ }^{175}$, N.A. Styles ${ }^{41}$, D.A. Soh ${ }^{151, w}$, D. Su ${ }^{143}$, HS. Subramania ${ }^{2}$, A. Succurro ${ }^{11}$, Y. Sugaya ${ }^{116}$, C. Suhr ${ }^{106}$, K. Suita ${ }^{66}$, M. Suk ${ }^{126}$, V.V. Sulin ${ }^{94}$, S. Sultansoy ${ }^{3 d}$, T. Sumida ${ }^{67}$, X. Sun ${ }^{55}$, J.E. Sundermann ${ }^{48}$, K. Suruliz ${ }^{139}$, G. Susinno ${ }^{36 a, 36 b}$, M.R. Sutton ${ }^{149}$, Y. Suzuki ${ }^{65}$, Y. Suzuki ${ }^{66}$, M. Svatos ${ }^{125}$, S. Swedish ${ }^{168}$, I. Sykora ${ }^{14 a}$, T. Sykora ${ }^{126}$, J. Sánchez ${ }^{167}$, D. Ta ${ }^{105}$, K. Tackmann ${ }^{41}$, A. Taffard ${ }^{163}$, R. Tafirout ${ }^{159 a}$, N. Taiblum ${ }^{153}$, Y. Takahashi ${ }^{101}$, H. Takai $^{24}$, R. Takashima $^{68}$, H. Takeda ${ }^{66}$, T. Takeshita ${ }^{140}$, Y. Takubo ${ }^{65}$, M. Talby ${ }^{83}$, A. Talyshev ${ }^{107, f}$, M.C. Tamsett ${ }^{24}$, J. Tanaka ${ }^{155}$, R. Tanaka ${ }^{15}$, S. Tanaka ${ }^{131}$, 
S. Tanaka ${ }^{65}$, A.J. Tanasijczuk ${ }^{142}$, K. Tani ${ }^{66}$, N. Tannoury ${ }^{83}$, S. Tapprogge ${ }^{81}$, D. Tardif $^{158}$, S. Tarem ${ }^{152}$, F. Tarrade ${ }^{28}$, G.F. Tartarelli ${ }^{89}$, P. Tas ${ }^{126}$, M. Tasevsky ${ }^{125}$, E. Tassi ${ }^{36 a, 36 b}$, M. Tatarkhanov ${ }^{14}$, Y. Tayalati ${ }^{135 d}$, C. Taylor ${ }^{77}$, F.E. Taylor $^{92}$, G.N. Taylor ${ }^{86}$, W. Taylor ${ }^{159 b}$, M. Teinturier ${ }^{15}$, M. Teixeira Dias Castanheira ${ }^{75}$, P. Teixeira-Dias ${ }^{76}$, K.K. Temming $^{48}$, H. Ten Kate ${ }^{29}$, P.K. Teng ${ }^{151}$, S. Terada ${ }^{65}$, K. Terashi ${ }^{155}$, J. Terron ${ }^{80}$, M. Testa ${ }^{47}$, R.J. Teuscher ${ }^{158, k}$, J. Therhaag $^{20}$, T. Theveneaux-Pelzer ${ }^{78}$, S. Thoma ${ }^{48}$, J.P. Thomas $^{17}$, E.N. Thompson ${ }^{34}$, P.D. Thompson ${ }^{17}$, P.D. Thompson ${ }^{158}$, A.S. Thompson ${ }^{53}$, L.A. Thomsen ${ }^{35}$, E. Thomson ${ }^{120}$, M. Thomson ${ }^{27}$, R.P. $\operatorname{Thun}^{87}$, F. $\operatorname{Tian}^{34}$, M.J. Tibbetts ${ }^{14}$, T. Tic ${ }^{125}$, V.O. Tikhomirov ${ }^{94}$, Y.A. Tikhonov ${ }^{107, f}$, S. Timoshenko ${ }^{96}$, P. Tipton ${ }^{176}$, F.J. Tique Aires Viegas ${ }^{29}$, S. Tisserant ${ }^{83}$, T. Todorov $^{4}$, S. Todorova-Nova ${ }^{161}$, B. Toggerson ${ }^{163}$, J. Tojo ${ }^{69}$, S. Tokár ${ }^{144 a}$, K. Tokunaga ${ }^{66}$, K. Tokushuku ${ }^{65}$, K. Tollefson ${ }^{88}$, M. Tomoto $^{101}$, L. Tompkins ${ }^{30}$, K. Toms ${ }^{103}$, A. Tonoyan ${ }^{13}$, C. Topfel ${ }^{16}$, N.D. Topilin ${ }^{64}$, I. Torchiani ${ }^{29}$, E. Torrence ${ }^{114}$, H. Torres $^{78}$, E. Torró Pastor ${ }^{167}$, J. Toth ${ }^{83, \text { ad }}$, F. Touchard ${ }^{83}$, D.R. Tovey ${ }^{139}$, T. Trefzger ${ }^{174}$, L. Tremblet $^{29}$, A. Tricoli ${ }^{29}$, I.M. Trig-

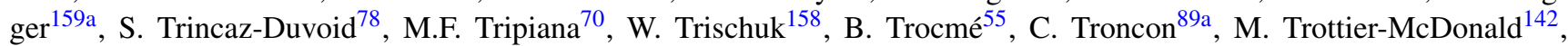
M. Trzebinski ${ }^{38}$, A. Trzupek ${ }^{38}$, C. Tsarouchas ${ }^{29}$, J.C-L. Tseng ${ }^{118}$, M. Tsiakiris ${ }^{105}$, P.V. Tsiareshka ${ }^{90}$, D. Tsionou ${ }^{4, \text { ah }}$, G. Tsipolitis ${ }^{9}$, V. Tsiskaridze ${ }^{48}$, E.G. Tskhadadze ${ }^{51 a}$, I.I. Tsukerman ${ }^{95}$, V. Tsulaia ${ }^{14}$, J.-W. Tsung ${ }^{20}$, S. Tsuno ${ }^{65}$, D. Tsybychev ${ }^{148}$, A. Tua ${ }^{139}$, A. Tudorache ${ }^{25 a}$, V. Tudorache ${ }^{25 a}$, J.M. Tuggle ${ }^{30}$, M. Turala ${ }^{38}$, D. Turecek ${ }^{127}$, I. Turk Cakir ${ }^{3 e}$,

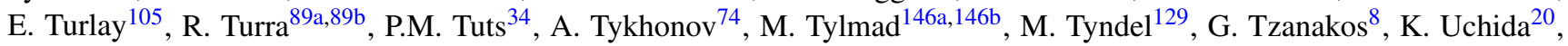

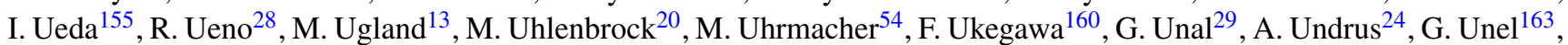
Y. Unno ${ }^{65}$, D. Urbaniec ${ }^{34}$, G. Usai ${ }^{7}$, M. Uslenghi ${ }^{119 a, 119 b}$, L. Vacavant ${ }^{83}$, V. Vacek ${ }^{127}$, B. Vachon ${ }^{85}$, S. Vahsen ${ }^{14}$, J. Valenta $^{125}$, P. Valente ${ }^{132 a}$, S. Valentinetti ${ }^{19 a, 19 b}$, S. Valkar ${ }^{126}$, E. Valladolid Gallego ${ }^{167}$, S. Vallecorsa ${ }^{152}$, J.A. Valls Ferrer ${ }^{167}$, H. van der Graaf ${ }^{105}$, E. van der Kraaij ${ }^{105}$, R. Van Der Leeuw ${ }^{105}$, E. van der Poel ${ }^{105}$, D. van der Ster $^{29}$, N. van Eldik ${ }^{29}$, P. van Gemmeren ${ }^{5}$, I. van Vulpen ${ }^{105}$, M. Vanadia ${ }^{99}$, W. Vandelli ${ }^{29}$, A. Vaniachine ${ }^{5}$, P. Vankov $^{41}$, F. Vannucci ${ }^{78}$, R. Vari $^{132 a}$, T. Varol ${ }^{84}$, D. Varouchas ${ }^{14}$, A. Vartapetian ${ }^{7}$, K.E. Varvell ${ }^{150}$, V.I. Vassilakopoulos ${ }^{56}$, F. Vazeille ${ }^{33}$, T. Vazquez Schroeder ${ }^{54}$, G. Vegni ${ }^{89 a, 89 b}$, J.J. Veillet ${ }^{115}$, F. Veloso ${ }^{124 a}$, R. Veness ${ }^{29}$, S. Veneziano ${ }^{132 a}$, A. Ventura ${ }^{72 a, 72 b}$, D. Ventura ${ }^{84}$, M. Venturi ${ }^{48}$, N. Venturi ${ }^{158}$, V. Vercesi ${ }^{119 a}$, M. Verducci ${ }^{138}$, W. Verkerke ${ }^{105}$, J.C. Vermeulen ${ }^{105}$, A. Vest ${ }^{43}$, M.C. Vetterli ${ }^{142, d}$, I. Vichou $^{165}$, T. Vickey ${ }^{145 b, a i}$, O.E. Vickey Boeriu ${ }^{145 b}$, G.H.A. Viehhauser ${ }^{118}$, S. Viel ${ }^{168}$, M. Villa ${ }^{19 a, 19 b}$, M. Villaplana Perez ${ }^{167}$, E. Vilucchi ${ }^{47}$, M.G. Vincter ${ }^{28}$, E. Vinek ${ }^{29}$, V.B. Vinogradov ${ }^{64}$, M. Virchaux ${ }^{136,{ }^{*}, \text { J. Virzi }^{14} \text {, O. Vitells }}{ }^{172}$, M. Viti ${ }^{41}$, I. Vivarelli $^{48}$, F. Vives Vaque ${ }^{2}$, S. Vlachos ${ }^{9}$, D. Vladoiu ${ }^{98}$, M. Vlasak ${ }^{127}$, A. Vogel ${ }^{20}$, P. Vokac ${ }^{127}$, G. Volpi ${ }^{47}$, M. Volpi ${ }^{86}$, G. Volpini ${ }^{89 a}$, H. von der Schmitt ${ }^{99}$, J. von Loeben ${ }^{99}$, H. von Radziewski ${ }^{48}$, E. von Toerne ${ }^{20}$, V. Vorobel ${ }^{126}$, V. Vorwerk $^{11}$, M. $\operatorname{Vos}^{167}$, R. Voss ${ }^{29}$, T.T. Voss ${ }^{175}$, J.H. Vossebeld ${ }^{73}$, N. Vranjes ${ }^{136}$, M. Vranjes Milosavljevic ${ }^{105}$, V. Vrba ${ }^{125}$, M. Vreeswijk ${ }^{105}$, T. Vu $\mathrm{Anh}^{48}$, R. Vuillermet ${ }^{29}$, I. Vukotic ${ }^{115}$, W. Wagner ${ }^{175}$, P. Wagner ${ }^{120}$, H. Wahlen ${ }^{175}$, S. Wahrmund ${ }^{43}$, J. Wakabayashi ${ }^{101}$, S. Walch ${ }^{87}$, J. Walder ${ }^{71}$, R. Walker ${ }^{98}$, W. Walkowiak ${ }^{141}$, R. Wall ${ }^{176}$, P. Waller ${ }^{73}$, C. Wang ${ }^{44}$, H. Wang ${ }^{173}$, H. Wang ${ }^{32 b, a j}$, J. Wang ${ }^{151}$, J. Wang ${ }^{55}$, R. Wang ${ }^{103}$, S.M. Wang ${ }^{151}$, T. Wang ${ }^{20}$, A. Warburton ${ }^{85}$, C.P. Ward ${ }^{27}$, M. Warsinsky ${ }^{48}$, A. Washbrook ${ }^{45}$, C. Wasicki ${ }^{41}$, P.M. Watkins ${ }^{17}$, A.T. Watson ${ }^{17}$, I.J. Watson ${ }^{150}$, M.F. Watson ${ }^{17}$, G. Watts ${ }^{138}$, S. Watts ${ }^{82}$, A.T. Waugh ${ }^{150}$, B.M. Waugh ${ }^{77}$, M. Weber ${ }^{129}$, M.S. Weber ${ }^{16}$, P. Weber ${ }^{54}$, A.R. Weidberg ${ }^{118}$, P. Weigell ${ }^{99}$, J. Weingarten ${ }^{54}$, C. Weiser ${ }^{48}$, H. Wellenstein ${ }^{22}$, P.S. Wells ${ }^{29}$, T. Wenaus ${ }^{24}$, D. Wendland ${ }^{15}$, Z. Weng ${ }^{151, w}$, T. Wengler ${ }^{29}$, S. Wenig ${ }^{29}$, N. Wermes $^{20}$, M. Werner ${ }^{48}$, P. Werner ${ }^{29}$, M. Werth ${ }^{163}$, M. Wessels ${ }^{58 a}$, J. Wetter ${ }^{161}$, C. Weydert ${ }^{55}$, K. Whalen ${ }^{28}$, S.J. WheelerEllis $^{163}$, A. White ${ }^{7}$, M.J. White ${ }^{86}$, S. White ${ }^{122 a, 122 b}$, S.R. Whitehead ${ }^{118}$, D. Whiteson ${ }^{163}$, D. Whittington ${ }^{60}$, F. Wicek ${ }^{115}$, D. Wicke $^{175}$, F.J. Wickens ${ }^{129}$, W. Wiedenmann ${ }^{173}$, M. Wielers ${ }^{129}$, P. Wienemann ${ }^{20}$, C. Wiglesworth ${ }^{75}$, L.A.M. WiikFuchs $^{48}$, P.A. Wijeratne ${ }^{77}$, A. Wildauer ${ }^{167}$, M.A. Wildt ${ }^{41, s}$, I. Wilhelm ${ }^{126}$, H.G. Wilkens ${ }^{29}$, J.Z. Will ${ }^{98}$, E. Williams ${ }^{34}$, H.H. Williams ${ }^{120}$, W. Willis ${ }^{34}$, S. Willocq ${ }^{84}$, J.A. Wilson ${ }^{17}$, M.G. Wilson ${ }^{143}$, A. Wilson ${ }^{87}$, I. Wingerter-Seez ${ }^{4}$, S. Winkelmann $^{48}$, F. Winklmeier ${ }^{29}$, M. Wittgen ${ }^{143}$, M.W. Wolter ${ }^{38}$, H. Wolters ${ }^{124 a, h}$, W.C. Wong ${ }^{40}$, G. Wooden ${ }^{87}$, B.K. Wosiek ${ }^{38}$, J. Wotschack ${ }^{29}$, M.J. Woudstra ${ }^{82}$, K.W. Wozniak ${ }^{38}$, K. Wraight ${ }^{53}$, C. Wright ${ }^{53}$, M. Wright ${ }^{53}$, B. Wrona ${ }^{73}$, S.L. Wu ${ }^{173}$, X. $\mathrm{Wu}^{49}$, Y. Wu ${ }^{32 b, a k}$, E. Wulf ${ }^{34}$, B.M. Wynne ${ }^{45}$, S. Xella ${ }^{35}$, M. Xiao ${ }^{136}$, S. Xie ${ }^{48}$, C. Xu ${ }^{32 b, z}$, D. Xu ${ }^{139}$, B. Yabsley ${ }^{150}$, S. Yacoob ${ }^{145 b}$, M. Yamada ${ }^{65}$, H. Yamaguchi ${ }^{155}$, A. Yamamoto ${ }^{65}$, K. Yamamoto ${ }^{63}$, S. Yamamoto ${ }^{155}$, T. Yamamura ${ }^{155}$, T. Yamanaka $^{155}$, J. Yamaoka ${ }^{44}$, T. Yamazaki ${ }^{155}$, Y. Yamazaki ${ }^{66}$, Z. Yan ${ }^{21}$, H. Yang ${ }^{87}$, U.K. Yang ${ }^{82}$, Y. Yang ${ }^{60}$, Z. Yang ${ }^{146 a, 146 b}$, S. Yanush ${ }^{11}$, L. Yao ${ }^{32 a}$, Y. Yao ${ }^{14}$, Y. Yasu ${ }^{65}$, G.V. Ybeles Smit ${ }^{130}$, J. Ye $^{39}$, S. Ye ${ }^{24}$, M. Yilmaz ${ }^{3 c}$, R. Yoosoofmiya ${ }^{123}$,

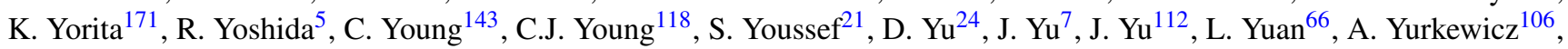
B. Zabinski ${ }^{38}$, R. Zaidan ${ }^{62}$, A.M. Zaitsev ${ }^{128}$, Z. Zajacova ${ }^{29}$, L. Zanello ${ }^{132 a, 132 b}$, A. Zaytsev ${ }^{107}$, C. Zeitnitz ${ }^{175}$, M. Zeman ${ }^{125}$, A. Zemla ${ }^{38}$, C. Zendler ${ }^{20}$, O. Zenin ${ }^{128}$, T. Ženišs ${ }^{14 a}$, Z. Zinonos ${ }^{122 a, 122 b}$, S. Zenz ${ }^{14}$, D. Zerwas ${ }^{115}$, G. Zevi della Porta ${ }^{57}$, Z. Zhan ${ }^{32 d}$, D. Zhang ${ }^{32 \text { baj }}$, H. Zhang ${ }^{88}$, J. Zhang ${ }^{5}$, X. Zhang ${ }^{32 d}$, Z. Zhang ${ }^{115}$, L. Zhao ${ }^{108}$, T. Zhao ${ }^{138}$, Z. Zhao ${ }^{32 b}$, A. Zhemchugov $^{64}$, J. Zhong ${ }^{118}$, B. Zhou ${ }^{87}$, N. Zhou ${ }^{163}$, Y. Zhou ${ }^{151}$, C.G. Zhu ${ }^{32 d}$, H. Zhu' ${ }^{41}$, J. Zhu ${ }^{87}$, Y. Zhu ${ }^{32 b}$, X. Zhuang ${ }^{98}$, V. Zhuravlov ${ }^{99}$, D. Zieminska ${ }^{60}$, N.I. Zimin ${ }^{64}$, R. Zimmermann ${ }^{20}$, S. Zimmermann ${ }^{20}$, S. Zimmermann ${ }^{48}$, M. Ziolkowski ${ }^{141}$, R. Zitoun ${ }^{4}$, L. Živković ${ }^{34}$, V.V. Zmouchko ${ }^{128, *}$, G. Zobernig ${ }^{173}$, A. Zoccoli ${ }^{19 a, 19 b}$, M. zur Nedden ${ }^{15}$, V. Zutshi ${ }^{106}$, L. Zwalinski $^{29}$ 
${ }^{1}$ University at Albany, Albany NY, United States of America

${ }^{2}$ Department of Physics, University of Alberta, Edmonton AB, Canada

${ }^{3(a)}$ Department of Physics, Ankara University, Ankara; ${ }^{(b)}$ Department of Physics, Dumlupinar University, Kutahya;

${ }^{(c)}$ Department of Physics, Gazi University, Ankara; ${ }^{(d)}$ Division of Physics, TOBB University of Economics and

Technology, Ankara; ${ }^{(e)}$ Turkish Atomic Energy Authority, Ankara, Turkey

${ }^{4}$ LAPP, CNRS/IN2P3 and Université de Savoie, Annecy-le-Vieux, France

${ }^{5}$ High Energy Physics Division, Argonne National Laboratory, Argonne IL, United States of America

${ }^{6}$ Department of Physics, University of Arizona, Tucson AZ, United States of America

${ }^{7}$ Department of Physics, The University of Texas at Arlington, Arlington TX, United States of America

${ }^{8}$ Physics Department, University of Athens, Athens, Greece

${ }^{9}$ Physics Department, National Technical University of Athens, Zografou, Greece

${ }^{10}$ Institute of Physics, Azerbaijan Academy of Sciences, Baku, Azerbaijan

${ }^{11}$ Institut de Física d'Altes Energies and Departament de Física de la Universitat Autònoma de Barcelona and ICREA, Barcelona, Spain

12(a) Institute of Physics, University of Belgrade, Belgrade; (b) Vinca Institute of Nuclear Sciences, University of Belgrade, Belgrade, Serbia

${ }^{13}$ Department for Physics and Technology, University of Bergen, Bergen, Norway

${ }^{14}$ Physics Division, Lawrence Berkeley National Laboratory and University of California, Berkeley CA, United States of America

${ }^{15}$ Department of Physics, Humboldt University, Berlin, Germany

${ }^{16}$ Albert Einstein Center for Fundamental Physics and Laboratory for High Energy Physics, University of Bern, Bern, Switzerland

${ }^{17}$ School of Physics and Astronomy, University of Birmingham, Birmingham, United Kingdom

${ }^{18(a)}$ Department of Physics, Bogazici University, Istanbul; ${ }^{(b)}$ Division of Physics, Dogus University, Istanbul;

(c) Department of Physics Engineering, Gaziantep University, Gaziantep; ${ }^{(d)}$ Department of Physics, Istanbul Technical University, Istanbul, Turkey

19(a) INFN Sezione di Bologna; (b) Dipartimento di Fisica, Università di Bologna, Bologna, Italy

${ }^{20}$ Physikalisches Institut, University of Bonn, Bonn, Germany

${ }^{21}$ Department of Physics, Boston University, Boston MA, United States of America

${ }^{22}$ Department of Physics, Brandeis University, Waltham MA, United States of America

23(a) Universidade Federal do Rio De Janeiro COPPE/EE/IF, Rio de Janeiro; ${ }^{(b)}$ Federal University of Juiz de Fora (UFJF), Juiz de Fora; ${ }^{(c)}$ Federal University of Sao Joao del Rei (UFSJ), Sao Joao del Rei; ${ }^{(d)}$ Instituto de Fisica, Universidade de Sao Paulo, Sao Paulo, Brazil

${ }^{24}$ Physics Department, Brookhaven National Laboratory, Upton NY, United States of America

${ }^{25(a)}$ National Institute of Physics and Nuclear Engineering, Bucharest; ${ }^{(b)}$ University Politehnica Bucharest, Bucharest;

${ }^{(c)}$ West University in Timisoara, Timisoara, Romania

${ }^{26}$ Departamento de Física, Universidad de Buenos Aires, Buenos Aires, Argentina

${ }^{27}$ Cavendish Laboratory, University of Cambridge, Cambridge, United Kingdom

${ }^{28}$ Department of Physics, Carleton University, Ottawa ON, Canada

${ }^{29} \mathrm{CERN}$, Geneva, Switzerland

${ }^{30}$ Enrico Fermi Institute, University of Chicago, Chicago IL, United States of America

31(a) Departamento de Física, Pontificia Universidad Católica de Chile, Santiago; ${ }^{(b)}$ Departamento de Física, Universidad

Técnica Federico Santa María, Valparaíso, Chile

32(a) Institute of High Energy Physics, Chinese Academy of Sciences, Beijing; ${ }^{(b)}$ Department of Modern Physics,

University of Science and Technology of China, Anhui; ${ }^{(c)}$ Department of Physics, Nanjing University, Jiangsu; ${ }^{(d)}$ School of Physics, Shandong University, Shandong, China

${ }^{33}$ Laboratoire de Physique Corpusculaire, Clermont Université and Université Blaise Pascal and CNRS/IN2P3, Aubiere Cedex, France

${ }^{34}$ Nevis Laboratory, Columbia University, Irvington NY, United States of America

${ }^{35}$ Niels Bohr Institute, University of Copenhagen, Kobenhavn, Denmark

36(a) INFN Gruppo Collegato di Cosenza, Cosenza; ${ }^{(\mathrm{b})}$ Dipartimento di Fisica, Università della Calabria, Arcavata di Rende, Italy

${ }^{37}$ AGH University of Science and Technology, Faculty of Physics and Applied Computer Science, Krakow, Poland 
${ }^{38}$ The Henryk Niewodniczanski Institute of Nuclear Physics, Polish Academy of Sciences, Krakow, Poland

${ }^{39}$ Physics Department, Southern Methodist University, Dallas TX, United States of America

${ }^{40}$ Physics Department, University of Texas at Dallas, Richardson TX, United States of America

${ }^{41}$ DESY, Hamburg and Zeuthen, Germany

${ }^{42}$ Institut für Experimentelle Physik IV, Technische Universität Dortmund, Dortmund, Germany

${ }^{43}$ Institut für Kern- und Teilchenphysik, Technical University Dresden, Dresden, Germany

${ }^{44}$ Department of Physics, Duke University, Durham NC, United States of America

${ }^{45}$ SUPA - School of Physics and Astronomy, University of Edinburgh, Edinburgh, United Kingdom

${ }^{46}$ Fachhochschule Wiener Neustadt, Johannes Gutenbergstrasse 3, 2700 Wiener Neustadt, Austria

${ }^{47}$ INFN Laboratori Nazionali di Frascati, Frascati, Italy

${ }^{48}$ Fakultät für Mathematik und Physik, Albert-Ludwigs-Universität, Freiburg i.Br., Germany

${ }^{49}$ Section de Physique, Université de Genève, Geneva, Switzerland

50(a) INFN Sezione di Genova; ${ }^{(\mathrm{b})}$ Dipartimento di Fisica, Università di Genova, Genova, Italy

${ }^{51(a)}$ E. Andronikashvili Institute of Physics, Tbilisi State University, Tbilisi; ${ }^{(b)}$ High Energy Physics Institute, Tbilisi State University, Tbilisi, Georgia

${ }^{52}$ II Physikalisches Institut, Justus-Liebig-Universität Giessen, Giessen, Germany

${ }^{53}$ SUPA - School of Physics and Astronomy, University of Glasgow, Glasgow, United Kingdom

${ }^{54}$ II Physikalisches Institut, Georg-August-Universität, Göttingen, Germany

${ }^{55}$ Laboratoire de Physique Subatomique et de Cosmologie, Université Joseph Fourier and CNRS/IN2P3 and Institut

National Polytechnique de Grenoble, Grenoble, France

${ }^{56}$ Department of Physics, Hampton University, Hampton VA, United States of America

${ }^{57}$ Laboratory for Particle Physics and Cosmology, Harvard University, Cambridge MA, United States of America

${ }^{58(a)}$ Kirchhoff-Institut für Physik, Ruprecht-Karls-Universität Heidelberg, Heidelberg; ${ }^{(b)}$ Physikalisches Institut,

Ruprecht-Karls-Universität Heidelberg, Heidelberg; ${ }^{(c)}$ ZITI Institut für technische Informatik,

Ruprecht-Karls-Universität Heidelberg, Mannheim, Germany

${ }^{59}$ Faculty of Applied Information Science, Hiroshima Institute of Technology, Hiroshima, Japan

${ }^{60}$ Department of Physics, Indiana University, Bloomington IN, United States of America

${ }^{61}$ Institut für Astro- und Teilchenphysik, Leopold-Franzens-Universität, Innsbruck, Austria

${ }^{62}$ University of Iowa, Iowa City IA, United States of America

${ }^{63}$ Department of Physics and Astronomy, Iowa State University, Ames IA, United States of America

${ }^{64}$ Joint Institute for Nuclear Research, JINR Dubna, Dubna, Russia

${ }^{65}$ KEK, High Energy Accelerator Research Organization, Tsukuba, Japan

${ }^{66}$ Graduate School of Science, Kobe University, Kobe, Japan

${ }^{67}$ Faculty of Science, Kyoto University, Kyoto, Japan

${ }^{68}$ Kyoto University of Education, Kyoto, Japan

${ }^{69}$ Department of Physics, Kyushu University, Fukuoka, Japan

${ }^{70}$ Instituto de Física La Plata, Universidad Nacional de La Plata and CONICET, La Plata, Argentina

${ }^{71}$ Physics Department, Lancaster University, Lancaster, United Kingdom

${ }^{72(a)}$ INFN Sezione di Lecce; ${ }^{(b)}$ Dipartimento di Matematica e Fisica, Università del Salento, Lecce, Italy

${ }^{73}$ Oliver Lodge Laboratory, University of Liverpool, Liverpool, United Kingdom

${ }^{74}$ Department of Physics, Jožef Stefan Institute and University of Ljubljana, Ljubljana, Slovenia

${ }^{75}$ School of Physics and Astronomy, Queen Mary University of London, London, United Kingdom

${ }^{76}$ Department of Physics, Royal Holloway University of London, Surrey, United Kingdom

${ }^{77}$ Department of Physics and Astronomy, University College London, London, United Kingdom

${ }^{78}$ Laboratoire de Physique Nucléaire et de Hautes Energies, UPMC and Université Paris-Diderot and CNRS/IN2P3, Paris, France

${ }^{79}$ Fysiska institutionen, Lunds universitet, Lund, Sweden

${ }^{80}$ Departamento de Fisica Teorica C-15, Universidad Autonoma de Madrid, Madrid, Spain

${ }^{81}$ Institut für Physik, Universität Mainz, Mainz, Germany

${ }^{82} \mathrm{School}$ of Physics and Astronomy, University of Manchester, Manchester, United Kingdom

${ }^{83}$ CPPM, Aix-Marseille Université and CNRS/IN2P3, Marseille, France

${ }^{84}$ Department of Physics, University of Massachusetts, Amherst MA, United States of America

${ }^{85}$ Department of Physics, McGill University, Montreal QC, Canada 
${ }^{86}$ School of Physics, University of Melbourne, Victoria, Australia

${ }^{87}$ Department of Physics, The University of Michigan, Ann Arbor MI, United States of America

${ }^{88}$ Department of Physics and Astronomy, Michigan State University, East Lansing MI, United States of America

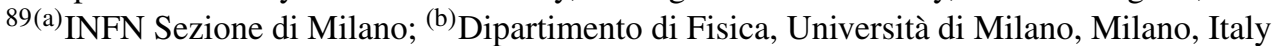

${ }^{90}$ B.I. Stepanov Institute of Physics, National Academy of Sciences of Belarus, Minsk, Republic of Belarus

${ }^{91}$ National Scientific and Educational Centre for Particle and High Energy Physics, Minsk, Republic of Belarus

${ }^{92}$ Department of Physics, Massachusetts Institute of Technology, Cambridge MA, United States of America

${ }^{93}$ Group of Particle Physics, University of Montreal, Montreal QC, Canada

${ }^{94}$ P.N. Lebedev Institute of Physics, Academy of Sciences, Moscow, Russia

${ }^{95}$ Institute for Theoretical and Experimental Physics (ITEP), Moscow, Russia

${ }^{96}$ Moscow Engineering and Physics Institute (MEPhI), Moscow, Russia

${ }^{97}$ Skobeltsyn Institute of Nuclear Physics, Lomonosov Moscow State University, Moscow, Russia

${ }^{98}$ Fakultät für Physik, Ludwig-Maximilians-Universität München, München, Germany

${ }^{99}$ Max-Planck-Institut für Physik (Werner-Heisenberg-Institut), München, Germany

${ }^{100}$ Nagasaki Institute of Applied Science, Nagasaki, Japan

${ }^{101}$ Graduate School of Science, Nagoya University, Nagoya, Japan

102(a) INFN Sezione di Napoli; ${ }^{(b)}$ Dipartimento di Scienze Fisiche, Università di Napoli, Napoli, Italy

${ }^{103}$ Department of Physics and Astronomy, University of New Mexico, Albuquerque NM, United States of America

${ }^{104}$ Institute for Mathematics, Astrophysics and Particle Physics, Radboud University Nijmegen/Nikhef, Nijmegen,

Netherlands

${ }^{105}$ Nikhef National Institute for Subatomic Physics and University of Amsterdam, Amsterdam, Netherlands

${ }^{106}$ Department of Physics, Northern Illinois University, DeKalb IL, United States of America

${ }^{107}$ Budker Institute of Nuclear Physics, SB RAS, Novosibirsk, Russia

${ }^{108}$ Department of Physics, New York University, New York NY, United States of America

${ }^{109}$ Ohio State University, Columbus OH, United States of America

${ }^{110}$ Faculty of Science, Okayama University, Okayama, Japan

${ }^{111}$ Homer L. Dodge Department of Physics and Astronomy, University of Oklahoma, Norman OK, United States of America

${ }^{112}$ Department of Physics, Oklahoma State University, Stillwater OK, United States of America

${ }^{113}$ Palacký University, RCPTM, Olomouc, Czech Republic

${ }^{114}$ Center for High Energy Physics, University of Oregon, Eugene OR, United States of America

${ }^{115}$ LAL, Université Paris-Sud and CNRS/IN2P3, Orsay, France

${ }^{116}$ Graduate School of Science, Osaka University, Osaka, Japan

${ }^{117}$ Department of Physics, University of Oslo, Oslo, Norway

${ }^{118}$ Department of Physics, Oxford University, Oxford, United Kingdom

119(a) INFN Sezione di Pavia; ${ }^{(b)}$ Dipartimento di Fisica, Università di Pavia, Pavia, Italy

${ }^{120}$ Department of Physics, University of Pennsylvania, Philadelphia PA, United States of America

${ }^{121}$ Petersburg Nuclear Physics Institute, Gatchina, Russia

122 (a) INFN Sezione di Pisa; ${ }^{(b)}$ Dipartimento di Fisica E. Fermi, Università di Pisa, Pisa, Italy

${ }^{123}$ Department of Physics and Astronomy, University of Pittsburgh, Pittsburgh PA, United States of America

124(a) Laboratorio de Instrumentacao e Fisica Experimental de Particulas - LIP, Lisboa, Portugal; ${ }^{(b)}$ Departamento de Fisica

Teorica y del Cosmos and CAFPE, Universidad de Granada, Granada, Spain

${ }^{125}$ Institute of Physics, Academy of Sciences of the Czech Republic, Praha, Czech Republic

${ }^{126}$ Faculty of Mathematics and Physics, Charles University in Prague, Praha, Czech Republic

${ }^{127}$ Czech Technical University in Prague, Praha, Czech Republic

${ }^{128}$ State Research Center Institute for High Energy Physics, Protvino, Russia

${ }^{129}$ Particle Physics Department, Rutherford Appleton Laboratory, Didcot, United Kingdom

${ }^{130}$ Physics Department, University of Regina, Regina SK, Canada

${ }^{131}$ Ritsumeikan University, Kusatsu, Shiga, Japan

132(a) INFN Sezione di Roma I; ${ }^{\left({ }^{b}\right)}$ Dipartimento di Fisica, Università La Sapienza, Roma, Italy

133(a) INFN Sezione di Roma Tor Vergata; ${ }^{(b)}$ Dipartimento di Fisica, Università di Roma Tor Vergata, Roma, Italy

134(a) INFN Sezione di Roma Tre; ${ }^{(b)}$ Dipartimento di Fisica, Università Roma Tre, Roma, Italy

${ }^{135(a)}$ Faculté des Sciences Ain Chock, Réseau Universitaire de Physique des Hautes Energies - Université Hassan II,

Casablanca; ${ }^{(b)}$ Centre National de l'Energie des Sciences Techniques Nucleaires, Rabat; ${ }^{(c)}$ Faculté des Sciences 
Semlalia, Université Cadi Ayyad, LPHEA Marrakech; ${ }^{(d)}$ Faculté des Sciences, Université Mohamed Premier and

LPTPM, Oujda; ${ }^{(e)}$ Faculté des sciences, Université Mohammed V-Agdal, Rabat, Morocco

${ }^{136}$ DSM/IRFU (Institut de Recherches sur les Lois Fondamentales de l'Univers), CEA Saclay (Commissariat a l'Energie

Atomique), Gif-sur-Yvette, France

${ }^{137}$ Santa Cruz Institute for Particle Physics, University of California Santa Cruz, Santa Cruz CA, United States of America

${ }^{138}$ Department of Physics, University of Washington, Seattle WA, United States of America

${ }^{139}$ Department of Physics and Astronomy, University of Sheffield, Sheffield, United Kingdom

${ }^{140}$ Department of Physics, Shinshu University, Nagano, Japan

${ }^{141}$ Fachbereich Physik, Universität Siegen, Siegen, Germany

${ }^{142}$ Department of Physics, Simon Fraser University, Burnaby BC, Canada

${ }^{143}$ SLAC National Accelerator Laboratory, Stanford CA, United States of America

${ }^{144(a)}$ Faculty of Mathematics, Physics \& Informatics, Comenius University, Bratislava; ${ }^{(b)}$ Department of Subnuclear

Physics, Institute of Experimental Physics of the Slovak Academy of Sciences, Kosice, Slovak Republic

${ }^{145(a)}$ Department of Physics, University of Johannesburg, Johannesburg; ${ }^{(b)}$ School of Physics, University of the

Witwatersrand, Johannesburg, South Africa

146(a) Department of Physics, Stockholm University; ${ }^{(b)}$ The Oskar Klein Centre, Stockholm, Sweden

${ }^{147}$ Physics Department, Royal Institute of Technology, Stockholm, Sweden

${ }^{148}$ Departments of Physics \& Astronomy and Chemistry, Stony Brook University, Stony Brook NY, United States of

America

${ }^{149}$ Department of Physics and Astronomy, University of Sussex, Brighton, United Kingdom

${ }^{150}$ School of Physics, University of Sydney, Sydney, Australia

${ }^{151}$ Institute of Physics, Academia Sinica, Taipei, Taiwan

${ }^{152}$ Department of Physics, Technion: Israel Institute of Technology, Haifa, Israel

${ }^{153}$ Raymond and Beverly Sackler School of Physics and Astronomy, Tel Aviv University, Tel Aviv, Israel

${ }^{154}$ Department of Physics, Aristotle University of Thessaloniki, Thessaloniki, Greece

${ }^{155}$ International Center for Elementary Particle Physics and Department of Physics, The University of Tokyo, Tokyo, Japan

${ }^{156}$ Graduate School of Science and Technology, Tokyo Metropolitan University, Tokyo, Japan

${ }^{157}$ Department of Physics, Tokyo Institute of Technology, Tokyo, Japan

${ }^{158}$ Department of Physics, University of Toronto, Toronto ON, Canada

159(a) TRIUMF, Vancouver BC; (b) Department of Physics and Astronomy, York University, Toronto ON, Canada

${ }^{160}$ Institute of Pure and Applied Sciences, University of Tsukuba, 1-1-1 Tennodai, Tsukuba, Ibaraki 305-8571, Japan

${ }^{161}$ Science and Technology Center, Tufts University, Medford MA, United States of America

${ }^{162}$ Centro de Investigaciones, Universidad Antonio Narino, Bogota, Colombia

${ }^{163}$ Department of Physics and Astronomy, University of California Irvine, Irvine CA, United States of America

164(a) INFN Gruppo Collegato di Udine, Udine; ${ }^{(b)}$ ICTP, Trieste; ${ }^{(c)}$ Dipartimento di Chimica, Fisica e Ambiente, Università di Udine, Udine, Italy

${ }^{165}$ Department of Physics, University of Illinois, Urbana IL, United States of America

${ }^{166}$ Department of Physics and Astronomy, University of Uppsala, Uppsala, Sweden

${ }^{167}$ Instituto de Física Corpuscular (IFIC) and Departamento de Física Atómica, Molecular y Nuclear and Departamento de Ingeniería Electrónica and Instituto de Microelectrónica de Barcelona (IMB-CNM), University of Valencia and CSIC, Valencia, Spain

${ }^{168}$ Department of Physics, University of British Columbia, Vancouver BC, Canada

${ }^{169}$ Department of Physics and Astronomy, University of Victoria, Victoria BC, Canada

${ }^{170}$ Department of Physics, University of Warwick, Coventry, United Kingdom

${ }^{171}$ Waseda University, Tokyo, Japan

${ }^{172}$ Department of Particle Physics, The Weizmann Institute of Science, Rehovot, Israel

${ }^{173}$ Department of Physics, University of Wisconsin, Madison WI, United States of America

${ }^{174}$ Fakultät für Physik und Astronomie, Julius-Maximilians-Universität, Würzburg, Germany

${ }^{175}$ Fachbereich C Physik, Bergische Universität Wuppertal, Wuppertal, Germany

${ }^{176}$ Department of Physics, Yale University, New Haven CT, United States of America

${ }^{177}$ Yerevan Physics Institute, Yerevan, Armenia

${ }^{178}$ Domaine scientifique de la Doua, Centre de Calcul CNRS/IN2P3, Villeurbanne Cedex, France

${ }^{a}$ Also at Laboratorio de Instrumentacao e Fisica Experimental de Particulas - LIP, Lisboa, Portugal 
${ }^{\mathrm{b}}$ Also at Faculdade de Ciencias and CFNUL, Universidade de Lisboa, Lisboa, Portugal

${ }^{\mathrm{c}}$ Also at Particle Physics Department, Rutherford Appleton Laboratory, Didcot, United Kingdom

${ }^{\mathrm{d} A l s o}$ at TRIUMF, Vancouver BC, Canada

eAlso at Department of Physics, California State University, Fresno CA, United States of America

${ }^{\mathrm{f}}$ Also at Novosibirsk State University, Novosibirsk, Russia

${ }^{g}$ Also at Fermilab, Batavia IL, United States of America

${ }^{\mathrm{h}}$ Also at Department of Physics, University of Coimbra, Coimbra, Portugal

${ }^{\mathrm{i}}$ Also at Department of Physics, UASLP, San Luis Potosi, Mexico

${ }^{j}$ Also at Università di Napoli Parthenope, Napoli, Italy

${ }^{\mathrm{k}}$ Also at Institute of Particle Physics (IPP), Canada

${ }^{1}$ Also at Department of Physics, Middle East Technical University, Ankara, Turkey

${ }^{m}$ Also at Louisiana Tech University, Ruston LA, United States of America

${ }^{\mathrm{n}}$ Also at Dep Fisica and CEFITEC of Faculdade de Ciencias e Tecnologia, Universidade Nova de Lisboa, Caparica, Portugal

${ }^{\circ}$ Also at Department of Physics and Astronomy, University College London, London, United Kingdom

pAlso at Group of Particle Physics, University of Montreal, Montreal QC, Canada

${ }^{\mathrm{q}}$ Also at Department of Physics, University of Cape Town, Cape Town, South Africa

${ }^{\mathrm{r}}$ Also at Institute of Physics, Azerbaijan Academy of Sciences, Baku, Azerbaijan

${ }^{\mathrm{s}}$ Also at Institut für Experimentalphysik, Universität Hamburg, Hamburg, Germany

${ }^{\mathrm{t}}$ Also at Manhattan College, New York NY, United States of America

"Also at School of Physics, Shandong University, Shandong, China

${ }^{v}$ Also at CPPM, Aix-Marseille Université and CNRS/IN2P3, Marseille, France

${ }^{\mathrm{w}}$ Also at School of Physics and Engineering, Sun Yat-sen University, Guanzhou, China

${ }^{x}$ Also at Academia Sinica Grid Computing, Institute of Physics, Academia Sinica, Taipei, Taiwan

${ }^{y}$ Also at Dipartimento di Fisica, Università La Sapienza, Roma, Italy

${ }^{z}$ Also at DSM/IRFU (Institut de Recherches sur les Lois Fondamentales de l'Univers), CEA Saclay (Commissariat a l'Energie Atomique), Gif-sur-Yvette, France

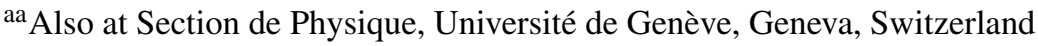

${ }^{a b}$ Also at Departamento de Fisica, Universidade de Minho, Braga, Portugal

${ }^{a c}$ Also at Department of Physics and Astronomy, University of South Carolina, Columbia SC, United States of America

${ }^{a d}$ Also at Institute for Particle and Nuclear Physics, Wigner Research Centre for Physics, Budapest, Hungary

${ }^{a e}$ Also at California Institute of Technology, Pasadena CA, United States of America

af Also at Institute of Physics, Jagiellonian University, Krakow, Poland

${ }^{\text {ag }}$ Also at LAL, Université Paris-Sud and CNRS/IN2P3, Orsay, France

${ }^{\text {ah }}$ Also at Department of Physics and Astronomy, University of Sheffield, Sheffield, United Kingdom

ai Also at Department of Physics, Oxford University, Oxford, United Kingdom

ajAlso at Institute of Physics, Academia Sinica, Taipei, Taiwan

${ }^{a k}$ Also at Department of Physics, The University of Michigan, Ann Arbor MI, United States of America

*Deceased 